\title{
Predicción de la Dirección de Variación del Precio de Una Acción De la Bolsa de Nueva York Usando Información de la Red Social Stocktwits Mediante Algoritmos de Minería de Datos y Aprendizaje Automático
}

\author{
Predicting Price Direction Variation of a Stock From The New York Stock Exchange \\ Using StockTwits Social Network Information and Data Mining \& Machine Learning \\ Algorithms
}

Andrés Felipe Rodriguez Perez. ${ }^{\mathrm{a}}$
andres.rodriguezp@usantotomas.edu.co

\author{
Robert Romero. ${ }^{\mathrm{b}}$ \\ grobert.romero@gmail.com
}

\section{Resumen}

El internet cambió la forma en que los agentes negocian activos en la bolsa, debido a la posibilidad de acceso inmediato a fuentes, artículos y estadísticas específicas para la acción que se desee. Sin embargo, estos no se encargan únicamente de demandar este tipo de fuentes; dado que generan grandes cantidades de información que contiene la opinión general sobre el mercado en un momento específico haciendo uso de redes sociales. Este trabajo estudia si esta información tiene capacidad predictiva sobre la variación de la dirección del precio de una activo transado en la Bolsa de Valores de Nueva York, valiéndose de herramientas de minería de datos y algoritmos de aprendizaje de máquina.

Palabras Claves: Bolsa de Valores de Nueva York, Precio del Activo, Minería de Datos, Aprendizaje Automático, Procesamiento del Lenguaje Natural, Análisis de Redes Sociales.

\begin{abstract}
Internet has changed the way traders trade in stock markets, due to the instant access to specific resources, articles and statistics about any stock. However, by using social networks; the traders took an important role in the generation of large ammounts of information in wich the main opinion about the market is contained. This work digs about if this information has any predictive power over the price direction variation of a stock traded in the New York Stock Exchange, using data mining techniques and machine learning algorithms.
\end{abstract}

keywords: New York Stock Exchange, Stock Price, Data Mining, Machine Learning, Natural Language Procesing, Social Network Analysis.

\footnotetext{
${ }^{a}$ Estudiante Estadística Universidad Santo Tomás

${ }^{\mathrm{b}}$ Estadístico. Profesor Facultad de Estadística Universidad Santo Tomás
} 


\section{Introducción}

La negociación diaria de activos en la bolsa de valores tiene un factor que la hace compleja e impredecible, la volatilidad. Esto genera mucha incertidumbre y cuando se trata de una decisión de inversión que involucra mucho capital las pérdidas pueden ser millonarias si no se logra un retorno aceptable. Además, los precios de los activos son bastante complejos de predecir debido a que los modelos tradicionales de series de tiempo y modelamiento de la volatilidad no tienen en cuenta las intenciones de los agentes involucrados en la oferta y la demanda, pues cada comprador y vendedor activo en el mercado cumple un rol en la determinación del precio del activo.

Sin embargo, predecir las acciones de cada comprador o vendedor potencial es sumamente complejo y no existe un protocolo de almacenamiento de datos relacionado con las intenciones de los agentes del mercado, estas se quedan en la cabeza de cada uno de ellos, influenciadas por la información disponible que tienen del mercado y el análisis técnico que realizan para tomar su decisión.

Afortunadamente, las redes sociales se han convertido en un insumo potencial en la determinación de una opinión general o reacción ante un hecho específico y existen trabajos recientes que demuestran que dicho "sentimiento" general puede ser tomado como aproximación de las intenciones de un agente en un mercado específico. (Asur \& Huberman, 2010) demostraron con éxito la capacidad predictiva que tiene el sentimiento general en twitter sobre un evento, en las ventas de taquilla valiéndose de redes neuronales. (Feldman, 2013) se encargó de discutir diferentes aplicaciones de la clasificación de sentimientos o extracción de información léxica de textos de redes sociales, entre ellas la capacidad de predecir cosas como los gustos musicales hasta los hábitos civiles de votación.

Internet está cambiando la forma en que los agentes del mercado de valores negocian, este brinda acceso inmediato a fuentes, artículos y estadísticas sobre lo que este desee buscar. Sin embargo, no solo demandan esta información sino que se han convertido en generadores masivos de la misma, cualquier persona con una conexión a Internet un computador y una cuenta de una red social es libre de generar opinión y discusión con miles de usuarios en tiempo real sobre cualquier tema.

En esta investigación se va a hacer énfasis específico en la red social "StockTwits", la cual existe para que los usuarios, inversionistas y aficionados compartan ideas acerca de los precios de las acciones, el comportamiento del mercado y la influencia de los factores microeconómicos y macroeconómicos externos sobre el mercado. Toda esta información es totalmente libre al público y la minería de datos sobre la misma es sencilla debido a que los textos contienen identificadores sobre el tema que tratan (un activo específico, tema o tendencia).

Siguiendo la biografiaría existente y la metodología propuesta por (Coyne et al, 2017); se propone un modelo de procesamiento del lenguaje natural para el etiquetado de los textos según su sentimiento y otra estrategia de aprovechamiento de la información de la red social, incluyendo otra manera de identificar usuarios influyentes ("smart users") y la relevancia de los comentarios por su numero de "likes". El objetivo es predecir la dirección del cambio en el precio de apertura y de cierre del activo valiéndose de esta información.

El texto se compone de 14 secciones, de la segunda a la octava se brinda un marco teórico y conceptual, junto con una revisión al estado del arte sobre los temas centrales de esta investigación incluyendo el desarrollo matemático de los modelos usados para la predicción del precio y el procesamiento de los textos. La novena sección da una breve referencia de como es el proceso de obtención de datos tanto de la red social como de los precios de los activos. La décima y onceava dan cuenta de la especificación del modelo de procesamiento de lenguaje natural y la metodología propuesta para la predicción de la dirección de variación del precio. En la doceava se muestran los resultados obtenidos y en la treceava las conclusiones y en la catorceava las referencias bibliográficas. 


\section{Procesamiento del lenguaje natural}

\section{1. ¿Que es el procesamiento del lenguaje natural?}

Según (Copestake, 2004) el Procesamiento del Lenguaje Natural o NLP por sus siglas en inglés, puede ser definido como el procesamiento automático (o semiautomático) del lenguaje humano. El término algunas veces toma definiciones más concretas, algunas en las cuales se excluye la obtención de la información e incluso la traducción a lenguaje de máquina para su aplicación. El NLP algunas veces es contrastado con "lingüística computacional", viéndose desde un campo más aplicado.

Esta autora afirma que NLP es esencialmente un campo multidisciplinario, esta relacionado muy de cerca con la lingüística, teniendo en cuenta que el grado en el que se relaciona con la teoría lingüística varía de forma considerable. Este también tiene una fuerte relación con la investigación en ciencia cognitiva, psicología, filosofía y matemáticas (especialmente lógica).

Debido a que involucra tantas disciplinas tiene innumerables campos de aplicación como el reconocimiento del habla, corrección automática, inteligencia artificial, por nombrar algunas. Gracias a esto ha tenido gran acogida dentro de varias industrias, que han revolucionado el tema.

Es por esto que autores más recientes como (Beysolow, 2018) hablan del NLP como un subcampo de la ciencia computacional o "Computer Science" que esta enfocado en enseñar a los computadores a entender el lenguaje de una forma "natural"; tal y como un humano lo entendería, para resolver tareas relacionadas con entender el sentimiento de un texto y generación de respuestas automatizadas a preguntas generadas por el usuario.

"NLP se ha convertido rápidamente en un campo envolvente dentro del cual sus aplicaciones representan una gran porción de los avances en Inteligencia Artificial” (Beysolow, 2018)

\subsection{Aproximaciones clásicas al procesamiento del lenguaje natural}

El enfoque tradicional del procesamiento natural del lenguaje se ha venido desarrollando al ver el proceso de análisis del lenguaje como una serie de fases o pasos (resumidos en la figura 1), reflejando las distinciones teóricas de la lingüística entre Sintaxis, Semántica y Pragmática (Dale, 2010). De manera más simple, esto implica analizar las frases de un texto primero en términos de su sintaxis, lo que deja ver una estructura que es más fácil de analizar en términos de semántica o significado literal, y luego se sigue con una etapa de análisis pragmático en el cual se determina el significado del texto según su contexto. Esta etapa final es entendida o tiene una fuerte relación con el discurso, y las dos etapas previas se relacionan únicamente con temas de significado.

Este intento de asociación entre las distinciones estratificadas de un texto (sintaxis, semántica, y pragmática) y la distinción específica entre frase versus discurso, algunas veces causa confusión al tratar con la resolución de problemas que implican procesamiento del lenguaje natural y es ampliamente reconocido que en términos reales no es tan simple separar el procesamiento del lenguaje en fases o "cajas". Sin embargo, esta separación sirve de ayuda pedagógica y comprende las bases arquitectónicas de modelos que hacen la tarea de el análisis natural del lenguaje sea manejable desde un punto de vista de ingeniería de software.

Dentro de este proceso se debe tener en cuenta un paso cero o paso inicial crucial proveniente de la necesidad combinar el estado del arte actual y el reto de tratar con datos reales de lenguaje. La etapa de "tokenización" o segmentación en frases (que incluye el pre procesamiento del texto) es necesaria debido a que los textos de lenguaje natural generalmente no están compuestos de frases cortas, concisas, bien estructuradas y bien delimitadas. 
En cierta manera, esta descomposición tan específica refleja el estado actual del conocimiento sobre procesamiento del lenguaje; debido a que se han desarrollado bastantes técnicas de tokenización, análisis léxico y sintáctico. Por otro lado no existen muchas enfocadas en el procesamiento a nivel de semántica o discurso del texto, demostrando el hecho de que lo conocido es la capa superficial de un texto, pero algo más profundo representa una abstracción difícil de precisar (Dale, 2010).

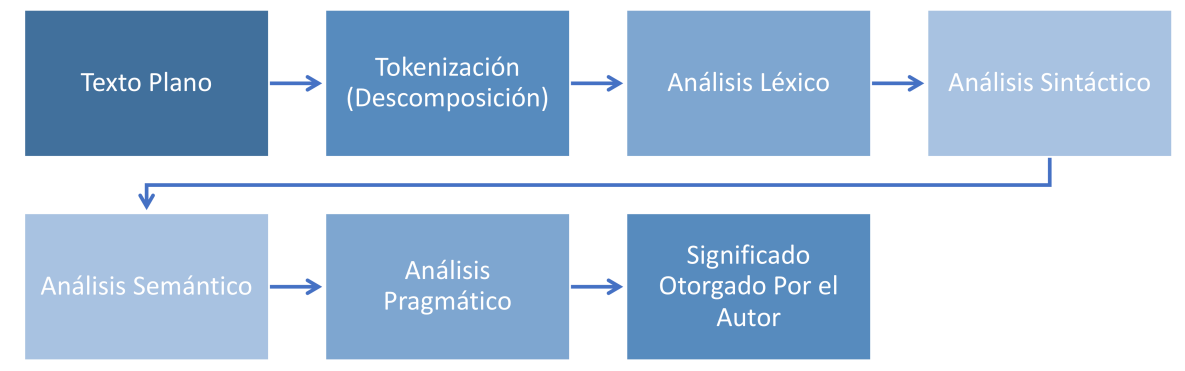

Figura 1: Etapas de análisis del NLP, Adaptado de: Indurkhya, Nitin, and Fred J. Damerau, eds. Handbook of natural language processing. Vol. 2. CRC Press, 2010.

\subsection{Aproximaciones empíricas y estadísticas al procesamiento del Lenguaje Natural: el auge del machine learning}

En los años ochenta pero especialmente sobre los noventa, se dio un resurgimiento importante de métodos empíricos y estadísticos aplicados al procesamiento automático del lenguaje natural, conocidos como "Corpus-based, statistical or empirical methods" o métodos estadísticos, empíricos basados en un "cuerpo" de textos (Marquéz, 2000).

Un corpus o cuerpo puede ser definido como una colección de textos auténticos legibles por una máquina (incluyendo transcripciones de datos hablados), los cuales son seleccionados (por muestreo o conocimiento previo del investigador) para ser representativos sobre un lenguaje natural particular o variedad de lenguaje (McEnery et al. 2006). El corpus juega un rol esencial en las investigaciones relacionadas con el NLP y también en un amplio rango de investigaciones lingüísticas.

La única particularidad de un corpus es que su construcción sigue de una necesidad específica de investigación o resolución de un problema, por lo que usar corpus pre construidos para resolver un problema diferente para el que fueron diseñados puede llevar a resultados inconclusos o equivocaciones. Sin embargo, el uso de este método se ha vuelto cada vez más popular debido a la disponibilidad de grandes cantidades de datos de texto provenientes de diferentes fuentes, niveles de anotación, lenguajes, entre otras, y a la mejora exponencial del rendimiento de las máquinas y programas disponibles permitiendo la automatización total o parcial de un sistema NLP usando métodos estadísticos entrenados sobre corpus que contienen grandes cantidades fuentes de lenguaje natural calificadas o no calificadas.

Esta línea de investigación en NLP dedicada al procesamiento masivo de datos textuales ha contribuido al desarrollo de varios métodos y técnicas con aplicaciones a una amplia variedad de problemas relacionados con la adquisición y entendimiento natural del lenguaje, incluyendo la extracción automática de conocimiento léxico, desambiguación léxica y estructural (etiquetado con base en el discurso, desambiguación del sentido de las palabras y desambiguación de la frase prepositiva), inferencia gramatical y análisis robusto, extracción y recuperación de información, resumen automático, traducción, por nombrar algunas (Marquéz, 2000).

La mayoría de los primeros métodos usados para la adquisición de lenguaje, aplicados por la comunidad del NLP fueron adaptados de la teoría de la estadística y la información. Como 
consecuencia de esta colaboración, se dio un avance significativo en el desarrollo y adaptación de técnicas estadísticas bien conocidas para resolver problemas particulares de modelamiento y procesamiento automático del lenguaje. Este progreso fue especialmente notable en tareas de bajo nivel como procesamiento del discurso y extracción de conocimiento léxico y desambiguación, pero se hicieron esfuerzos considerables en inferencia gramatical, análisis robusto y otras tareas de nivel semántico y de discurso pertenecientes al NLP.

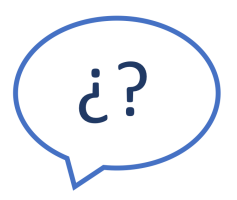

1. Identificación

Automática de

Discurso y

Conocimiento Léxico

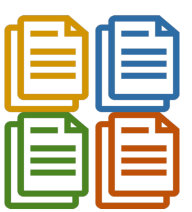

2. Construcción del Corpus (Para propósito específico)

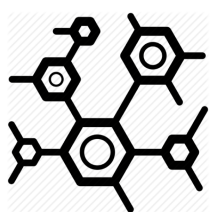

3. Modelos Estadísticos y de Machine Learning

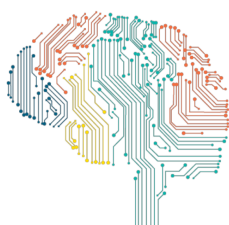

4. Extracción Automática de Conocimiento Léxico (AI)

Figura 2: Etapas de NLP Empírico y Estadístico, Elaboración Propia

Como se mencionó al inicio de este apartado, a inicios de los noventas pero particularmente en la actualidad, las aplicaciones de Aprendizaje del Lenguaje basadas en Aprendizaje de Máquina o "Machine Learning" (ML por sus siglas en inglés) han sido el foco de interés en la comunidad del NLP. Los problemas centrales tratados por el enfoque del Machine Learning son los que se centran en la desambiguación del lenguaje (apareciendo en todos los niveles del proceso de entendimiento del lenguaje). Estos métodos tienen la propiedad de ser particularmente apropiados debido a que pueden lidiar fácilmente con problemas de clasificación, un problema genérico de larga tradición dentro del área de la Inteligencia Artificial (AI por sus siglas en inglés), ampliamente tratada por la comunidad del ML.

Algunos de los métodos basados en ML incluyen una amplia variedad de paradigmas de aprendizaje simbólico inductivo como los árboles de decisión, discriminantes lineales de umbral, lógica inductiva, segmentación no supervisada, entre otras; y también un número significativo de aproximaciones subsimbólicas y conexionistas, como las redes neuronales y algoritmos genéticos (Marquéz, 2000).

Como menciona (Marquéz, 2000), el desarrollo reciente del NLP ha tendido a enfocarse en adaptar técnicas de Machine Learning para resolver casos específicos del Procesamiento del Lenguaje Natural, sin embargo, en este trabajo solo especificará la naturaleza lógica y teórica detrás de algunos métodos de ML, que serán usados para resolver el problema o pregunta que trata esta investigación.

\section{Análisis de redes sociales y mercado de valores}

\subsection{Mercados de valores}

Los mercados de valores son sistemas complejos, y no son gobernados por simples ecuaciones lineales o no lineales; los precios de los activos están influenciados por los compradores y vendedores en el mercado, de tal manera que; ambos establecen sus ofertas y los mecanismos de compensación del mercado (figura 3) juntan la demanda (compradores) con la oferta (vendedores) para determinar el precio (Kooijman, 2014). Por todo lo anterior, un modelo más acertado; para el comportamiento del mercado de valores, debería ser uno que tenga en cuenta la influencia de múltiples agentes, donde 
los agentes representen a los compradores y los vendedores. (LeBaron, 2006) y (E. Samanidou and Lux, 2007).

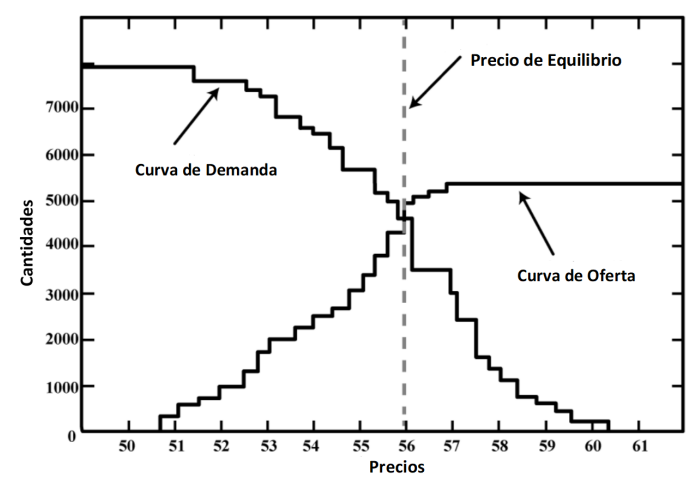

Figura 3: Precio de Mercado Establecido con la Curva de Oferta y Demanda, Recuperado de: Izenman, Alan Julian. Modern multivariate statistical techniques. Regression, classification and manifold learning (2008).

\subsection{Datos de StockTwits para predicciones en el mercado de valores}

Dado que es complejo determinar los movimientos de los actores en el mercado de valores y no existe una gran base de datos que almacene todas las intenciones y acciones de un potencial comprador, las investigaciones se han centrado en identificar si la información publicada en las redes sociales es una variable proxy con la suficiente capacidad predictiva para determinar las tendencias de compra y venta. Varios autores han evidenciado que, las redes sociales tienen un fuerte poder predictivo, por ejemplo, (Zhang, Fuehres \& Gloor, 2011) encontraron que el sentimiento general en Twitter tiene una correlación con como se moverá el mercado en conjunto. De las diferentes investigaciones y sus resultados surge la pregunta ¿Hay una correlación entre el sentimiento general de una red social y el precio de un activo en el mercado de valores?

(Oh \& Sheng, 2011) encontraron que los "micro blogs" o comentarios cortos, debido a su naturaleza de tiempo real y alto volumen tienen poder predictivo sobre movimientos del precio del activo en el futuro. Usando un clasificador de "bolsa de palabras" etiquetaron textos cortos según si expresaban sentimientos positivos o negativos. El sentimiento promedio fue usado para predecir el cambio en la dirección del precio del activo. Por otro lado Oliveira, Cortez \& Areal (2013) fueron más escépticos y criticaron el trabajo de Oh \& Sheng evidenciando que sus tamaños de muestra eran muy pequeños y asumir una correlación con estos era erróneo.

Esto no significó que se estableciera ausencia de poder predictivo en los datos de la red social Stocktwits, (Tsui) usó varios modelos; entre ellos un clasificador de bayes ("Naive Bayes"), obteniendo precisiones promedio por encima de lo deseado pero la variabilidad de resultados dependiendo del activo generó resultados inconclusos.

Otro tema importante para esta investigación se centra en los trabajos relacionados a la clasificación o etiquetado automático de tweets. Los trabajos anteriores basaron su predicción en modelos de bolsa de palabras, que pueden no ser tan efectivos extrayendo conocimiento léxico. (Nausheen, Kumar \& Amrutha) realizaron un estudio a fondo de 10 fuentes relacionadas en el área, muchas de ellas relacionadas con el mercado de valores, el tema principal fue la clasificación de sentimientos y como este ha mejorado notablemente en los últimos años y como los modelos más recientes de aprendizaje de máquina pueden lograr tasas muy altas de precisión al clasificar los textos en clusters dependiendo de la emoción que expresan. 
Las redes neuronales fueron introducidas por primera vez en el mercado de valores por (Kimoto, et al 1990). Su investigación, realizada antes del cambio de siglo; contiene una amplia gama de técnicas económicas y estadísticas como entrada. (Asur \& Huberman ,2010) fueron pioneros en usar predicciones basadas en redes neuronales en las redes sociales, pues lograron predecir con alta precisión ventas de taquillas basándose en el sentimiento individual de los tweets; trabajos que prueban que el aprendizaje profundo puede ser usado en redes sociales y en el mercado de valores.

\section{Redes neuronales artificiales}

\subsection{La neurona de McCuloch-Pitts}

La idea de una red neuronal "artificial" proviene del modelo de "máquina de computación" de McCullogh y Pitts (figura 4), quienes construyeron una abstracción del proceso que lleva la actividad neuronal en el cerebro humano. Este modelo, se compone de múltiples entradas (las dendritas) y una salida (el axón). Las entradas denotadas como $X_{1}, X_{2}, X_{3}, \ldots, X_{r}$ para las cuales cada una puede tener el valor de 0 ("apagado") o 1 ("encendido"), se suman para obtener la excitación total $U=\sum_{j} X_{j}$, y cuando $U$ es comparado con un valor de tolerancia $\theta$; y $U \geq \theta$ la salida de $Y$ es igual a uno y la neurona se activa para transmitir una nueva señal; de lo contrario $Y$ es cero y la neurona no transmite.

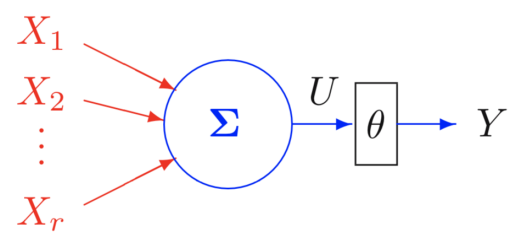

Figura 4: Neurona de McCuloch-Pits, Recuperado de: Izenman, Alan Julian. Modern multivariate statistical techniques. Regression, classification and manifold learning (2008).

Geométricamente, el espacio de entrada es un cubo unitario r-dimensional, y cada uno de los vértices $2^{r}$ del hipercubo es asociado con un valor específico de Y. Para un valor dado de $\theta$, la neurona divide el hipercubo en dos espacios de acuerdo al hiperplano $\sum_{j} X_{j}=\theta$; cuyos vértices con $Y=1$ pertenecen a un lado del hiperplano, y los de $Y=0$ en el otro lado.

\subsection{Perceptrón de capa simple}

(Donald O Hebb, 1949) en su libro "La Organización del Comportamiento" establece que en el nacimiento, todas las conexiones neuronales se encuentran distribuidas de manera aleatoria, y conforme pasa el tiempo estas conexiones se multiplican y se hacen más fuertes. Sin embargo, la idea más importante de su trabajo, derivada de un descubrimiento de (Lorente de Nó, 1944); radica en que el cerebro contiene circuitos cerrados de neuronas en los cuales se almacena la memoria en la corteza cerebral en forma de clusters sobrepuestos de miles de neuronas fuertemente conectadas.

La teoría de (Donald O Hebb, 1949), llevó al psicólogo (Frank Rosenblatt, 1994) a buscar maneras de refutarla o mejorarla. En el proceso, afirmó que las conexiones neuronales no son aleatorias y que los clusters de neuronas se auto-generan. Para demostrarla propuso un modelo "mínimamente restringido" al cual llamó "perceptrón simple" (figura 5). 


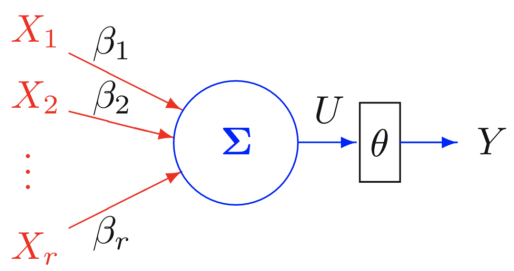

Figura 5: Perceptron Simple de Una Capa de Rosenblatt, Recuperado de: Izenman, Alan Julian. Modern multivariate statistical techniques. Regression, classification and manifold learning (2008).

El perceptrón simple es esencialmente una neurona de McCulloch-Pitts, pero ahora a cada una de las entradas $X_{i}$ le corresponde un valor real que representa el peso de la conexión $\beta_{i}, i=1,2, \ldots, r$, permitiendo así que las entradas $X_{1}, X_{2}, X_{3}, \ldots, X_{r}$ puedan ser binarias o reales. Valores positivos de $\beta$ reflejan sinapsis exitatorias y negativos representan sinapsis inhibitorias, siendo su magnitud la fuerza de la conexión.

Este modelo, más flexible que el de McCulloch-Pitts para imitar las conexiones neuronales, es esencialmente una suma ponderada de los valores de entrada $U=\sum_{j} \beta_{j} X_{j}$. $U$ es calculada, y el valor de salida es $Y=1$ solo si $U \geq \theta$, en donde $\theta$ es el valor de tolerancia; de lo contrario, $Y=0$

Una función $Y \in\{0,1\}$ es perceptrón-computable si, para un valor dado de $\theta$, existe un hiperplano que divide el espacio de entradas en dos partes, $R_{1}$ y $R_{0} . R_{1}$ corresponde a los puntos para los cuales $Y=1$ y $R_{0}$ a los puntos donde $Y=0$, Si los puntos en $R_{1}$ pueden ser separados de los de $R_{0}$ sin error en el hiperplano, se dice que son linealmente separables. Esta partición binaria del espacio de entrada (comparando $U$ con el parámetro $\theta$ de tolerancia), permite a un perceptrón predecir el miembro de una clase.

\subsection{Funciones de activación}

Dado $\boldsymbol{X}=\left(X_{1}, \ldots, X_{r}\right)^{T}$ un vector aleatorio r-dimensional de entradas, cada nodo de salida calcula un valor de activación usando una combinación lineal de las entradas mas una constante; siguiendo esta lógica, para cada l-ésimo nodo o neurona de salida, se calcula una l-ésima función lineal de activación (proceso resumido en la figura 6),

$$
U_{l}=\beta_{0 l}+\sum_{j=1}^{r} \beta_{j l} X_{j}=\beta_{0 l}+\boldsymbol{X}^{T} \beta_{l}
$$

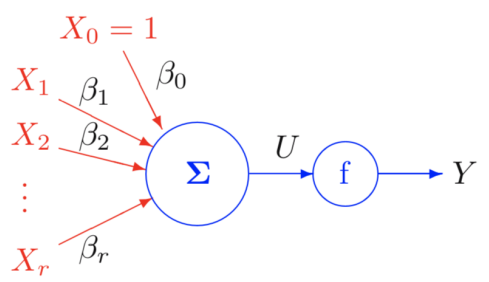

Figura 6: Perceptrón simple de una capa de Rosenblatt con unidad de función de activación, Recuperado de: Izenman, Alan Julian. Modern multivariate statistical techniques. Regression, classification and manifold learning (2008). 
donde $\beta_{0, l}$ es una constante (o sesgo) relacionada con la magnitud establecida en cada neurona para que esta se active, y $\beta_{l}=\left(\beta_{1 l}, \ldots, \beta_{r l}\right)^{T}$ es un vector r-dimensional de los pesos de sinapsis, para cada una de las $l=1, \ldots, s$ neuronas.

En notación matricial, la colección de $s$ funciones lineales de activación se puede denotar como

$$
U=\beta_{0}+B X
$$

donde $\boldsymbol{U}=\left(U_{1}, \ldots, U_{s}\right)^{T}, \beta_{0}=\left(\beta_{01}, \ldots, \beta_{0 s}\right)^{T}$ es un vector s-dimensional de sesgos, y $\boldsymbol{B}=$ $\left(\beta_{1}, \ldots, \beta_{s}\right)^{T}$ es una matriz $(s \mathrm{x} r)$ de pesos o ponderaciones de conexión. Los valores de activación, luego son filtrados a través de una función de tolerancia de activación $f\left(U_{l}\right)$ para formar el valor de salida del $l$-ésimo nodo, $l=1, \ldots, s$. En notación matricial,

$$
f(U)=f\left(\beta_{0}+B X\right)
$$

$\boldsymbol{f}=(f, \ldots, f)^{T}$ es un vector s-dimensional de funciones en el cual cada uno de sus elementos es la función $f$, y $f(U)=\left(f\left(U_{1}\right), \ldots, f\left(U_{s}\right)\right)^{T}$.

Una lista parcial de funciones de activación se muestra en el cuadro (1). Las más interesantes son las de tipo sigmoidal ("forma de $S$ ") como la logística y la tangente hiperbólica. Una función sigmoidal es una función $\sigma($.$) que tiene las siguientes propiedades: \sigma(u) \rightarrow 0$ cuando $u \rightarrow-\infty$ y $\sigma(u) \rightarrow 1$ cuando $u \rightarrow+\infty$. Una función sigmoidal $\sigma($.) es simétrica si $\sigma(u)+\sigma(-u)=1$ y asimétrica si $\sigma(u)+\sigma(-u)=0$.

Cuadro 1: Ejemplos de Funciones de Activación

\begin{tabular}{lcc}
\hline Función de Activación & $f(u)$ & Rango de Valores \\
\hline Identidad & $u$ & $\Re$ \\
Signo & $\operatorname{sign}(u)$ & $\{-1,+1\}$ \\
Límite & $I_{[u \geq 0]}$ & $\{0,1\}$ \\
Gausiana de Base Radial & $(2 \pi)^{-\frac{1}{2}} e^{-\frac{-u^{2}}{2}}$ & $\Re$ \\
Gausiana Acumulada (sigmoide) & $\sqrt{\frac{2}{\pi}} \int_{0}^{u} e^{-\frac{z^{2}}{2}} d z$ & $(0,1)$ \\
Logística (sigmoide) & $\left(1+e^{-u}\right)^{1}$ & $(0,1)$ \\
Tangente Hiperbólica (Sigmoide) & $\frac{\left(e^{u}-e^{-u}\right)}{\left(e^{u}+e^{-u}\right)}$ & $(-1,+1)$ \\
\end{tabular}

Recuperado de: Izenman, Alan Julian. Modern multivariate statistical techniques. Regression, classification and manifold learning (2008). 


\subsection{La regla de aprendizaje del perceptrón}

Para esta subsección, se hacen los siguientes cambios en la notación: $\beta \leftarrow\left(\beta_{0}, \boldsymbol{\beta}^{T}\right)^{T}$ y $\boldsymbol{X} \leftarrow\left(1, \boldsymbol{X}^{T}\right)^{T}$, donde $\boldsymbol{X}$ y $\boldsymbol{\beta}$ son ahora vectores $(r+1)$ dimensionales, por lo que podemos escribir $\beta_{0}+\boldsymbol{X}^{T} \boldsymbol{\beta}$ como $\boldsymbol{X}^{T} \boldsymbol{\beta}$. Para un caso simple de clasificación binaria, la variable de salida toma valores $Y= \pm 1$ dependiendo de si la neurona se activa o no. Por lo tanto la neurona se activará si $\boldsymbol{X}^{T} \boldsymbol{\beta} \geq 0$ y no lo hará si $\boldsymbol{X}^{T} \boldsymbol{\beta}<0$.

Suponiendo que $\boldsymbol{X}_{1}, \ldots, \boldsymbol{X}_{n}$ son copias independientes de $\boldsymbol{X}$, y son generadas a partir de las dos clases $\prod_{1}$ y $\prod_{2}$ y además que estas observaciones son linealmente separables. Esto sí, existe un vector $\beta^{*}$ de pesos de conexión tal que los vectores de observaciones que pertenecen a la clase $\prod_{1}$ pertenezcan al lado del hiperplano en el cual $\boldsymbol{X}^{T} \boldsymbol{\beta}^{*}=0$, mientras que los vectores de observaciones de la clase $\prod_{2}$ se encuentren en el otro lado del hiperplano.

Como regla de actualización, se usa un algoritmo de gradiente descendiente, que opera de manera secuencial en cada vector de entrada. Este algoritmo se relaciona con el aprendizaje en línea, en el cual el mecanismo de aprendizaje se adapta rápidamente para corregir los errores de clasificación conforme estos ocurren. Los vectores de entrada son examinados una vez y clasificados en una de las dos clases. Luego la clase verdadera es revelada y el procedimiento de clasificación es actualizado de acuerdo a esta.

El algoritmo procede reclasificando la copia $\left\{\boldsymbol{X}_{i}\right\}$, una a la vez, de tal manera que a la $h$-ésima iteración se esta tratando con $\boldsymbol{X}_{h}, h=1,2, \ldots$ estableciendo $\boldsymbol{X}_{0}=0$. El algoritmo calcula una secuencia $\left\{\boldsymbol{\beta}_{h}\right\}$ de pesos de conexión usando como valor inicial $\boldsymbol{\beta}_{0}=0$ y actualizándose bajo la siguiente regla:

1. Si en la $h$-ésima iteración, la versión actual de $\boldsymbol{\beta}_{h}$, clasifica correctamente $\boldsymbol{X}_{h}$, no se cambia $\boldsymbol{\beta}_{h}$ en la siguiente iteración; lo que significa establecer $\boldsymbol{\beta}_{h+1}=\boldsymbol{\beta}_{h}$ si algún $\boldsymbol{X}_{h}^{T} \boldsymbol{\beta}_{h} \geq 0 \mathrm{y}$ $\boldsymbol{X}_{h} \in \prod_{1}$, о $\boldsymbol{X}_{h}^{T} \boldsymbol{\beta}_{h}<0$ у $\boldsymbol{X}_{h} \in \prod_{2}$.

2. Si, por otro lado, la versión actual de $\boldsymbol{\beta}_{h}$, clasifica de manera errónea a $\boldsymbol{X}_{h}$, se actualizan los pesos de conexión de la siguiente manera: si $\boldsymbol{X}_{h}^{T} \boldsymbol{\beta}_{h} \geq 0$ pero $\boldsymbol{X}_{h} \in \prod_{2}$, entonces $\boldsymbol{\beta}_{h+1}=\boldsymbol{\beta}_{h}-\eta \boldsymbol{X}_{h}$; si $\boldsymbol{X}_{h}^{T} \boldsymbol{\beta}_{h}<0$ pero $\boldsymbol{X}_{h} \in \prod_{1}$, entonces se calcula $\boldsymbol{\beta}_{h+1}=\boldsymbol{\beta}_{h}+\eta \boldsymbol{X}_{h}$, donde $\eta>0$ es el parámetro de tasa de aprendizaje, la cual es independiente de la iteración $h$.

Este algoritmo es popularmente conocido como la regla de aprendizaje del perceptrón. Dado que el valor de $\eta$ es irrelevante (siempre se puede re escalar $\boldsymbol{X}_{h}$ y $\boldsymbol{\beta}_{h}$ ) se establece $\eta=1$ sin pérdida de generalidad.

\subsection{Teorema de convergencia del perceptrón}

De la regla de aprendizaje se tiene que $\beta_{h+1}=\sum_{i=1}^{h} \boldsymbol{X}_{i}$. Asumiendo que se tiene separabilidad lineal entre las dos clases y que la solución del vector $\beta^{*}$ existe, se define:

$$
A=\min _{\boldsymbol{X}_{i} \in \prod_{1}} \boldsymbol{X}_{i}^{T} \boldsymbol{\beta}^{*}, B=\operatorname{máx}_{\boldsymbol{X}_{* i} \in \prod_{1}}\left\|\boldsymbol{X}_{i}\right\|^{2}
$$

Transponiendo $\beta_{h+1}$ y luego multiplicando el resultado a través de $\beta^{*}$ se tiene:

$$
\beta_{h+1}^{T} \beta^{*}=\sum_{i=1}^{h} \boldsymbol{X}_{i}^{t} \beta^{*} \geq h A
$$

De la desigualdad de Cauchy-Schwartz, 


$$
\left(\beta_{h+1}^{T} \beta^{*}\right)^{2} \leq\left\|\beta_{h+1}^{T}\right\|^{2}\left\|\beta^{*}\right\|^{2}
$$

Substituyendo (5) en (6) se obtiene:

$$
\left\|\beta_{h+1}\right\|^{2} \geq \frac{h^{2} A^{2}}{\left\|\beta^{*}\right\|^{2}}
$$

Por lo tanto, la norma cuadrada del vector de pesos crece al menos de forma cuadrática con el número, $h$ de iteraciones.

Luego, considerando de nuevo la regla de actualización, $\boldsymbol{\beta}_{k+1}=\boldsymbol{\beta}_{k}+\boldsymbol{X}_{k}$, en la $k$-ésima iteración, en la cual $\boldsymbol{X}_{k} \in \prod_{1}, k=1,2, \ldots, h$. Entonces,

$$
\left\|\beta_{k+1}\right\|^{2}=\left\|\beta_{k}\right\|^{2}+\left\|X_{k}\right\|^{2}+2 X_{k}^{T} \beta_{k}
$$

Dado que $\boldsymbol{X}_{k}$ ha sido clasificado de manera incorrecta, $\boldsymbol{X}_{k}^{T}<0$. Resulta que,

$$
\left\|\beta_{k+1}\right\|^{2} \leq\left\|\beta_{k}\right\|^{2}+\left\|X_{k}\right\|^{2}
$$

de lo cual,

$$
\left\|\beta_{k+1}\right\|^{2}-\left\|\beta_{k}\right\|^{2} \leq\left\|X_{k}\right\|^{2}
$$

Sumando (10) sobre $k=1,2, \ldots, h$ se obtiene

$$
\left\|\beta_{h+1}\right\|^{2} \leq \sum_{k=1}^{h}\left\|X_{k}\right\|^{2} \leq h B .
$$

Por consiguiente, la norma cuadrada del vector de pesos crece por lo menos de manera lineal con el número, $h$, de iteraciones.

Para valores grandes de $h$, las desigualdades (7) y (11) se contradicen una a la otra. Por lo tanto $h$ no puede crecer sin límite. Es necesario encontrar un $h_{\max }$ que satisfaga

$$
\frac{h_{\max }^{2} A^{2}}{\left\|\beta^{*}\right\|^{2}}=h_{\max } B
$$

por lo cual,

$$
h_{\max }=\frac{B\left\|\beta^{*}\right\|^{2}}{A^{2}}
$$

Este resultado dado $\eta=1$ and $\boldsymbol{\beta}_{0}=0$, luego:

Para un problema de clasificación binario con clases linealmente separables, si un vector de solución $\beta^{*}$ existe, el algoritmo encontrará una solución en un número finito de, $h_{\max }$, iteraciones. 


\subsection{Perceptrones multicapa}

Dado que el perceptrón simple, como se mostró en las secciones anteriores; depende de que la variable de salida sea linealmente separable y que exista un número $h_{\max }$ de iteraciones (el cual es muy complejo de conocer dado que depende del vector de solución óptima $\beta^{*}$ ), es un modelo muy limitado para abordar casos de clasificación en los que no se cumplan estas condiciones. (Minsky \& Papert, 1969) sugirieron en sus trabajos que estas limitaciones se pueden superar apilando los perceptrones en estratificaciones o niveles y aplicando transformaciones no lineales a las variables de entrada ya ponderadas, sugerencias que se volvieron significativas con el aumento de la capacidad de procesamiento de los computadores y la aparición del algoritmo de "retropropagación".

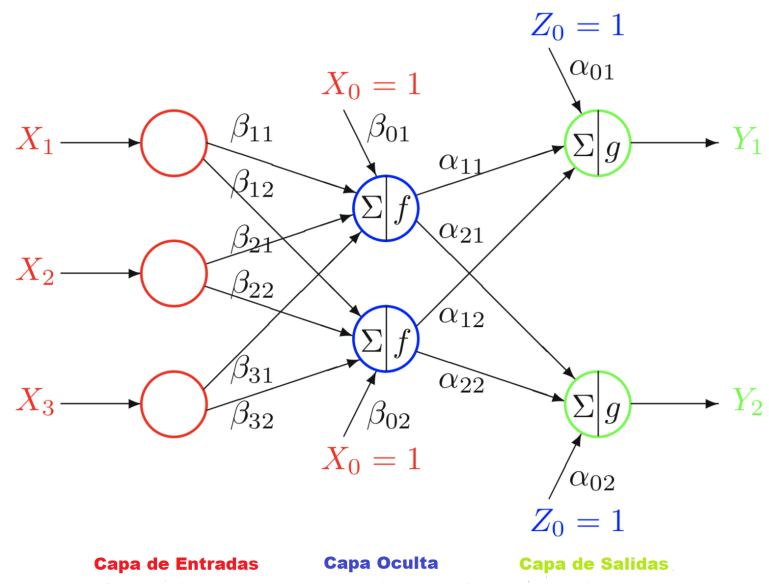

Figura 7: Perceptrón multicapa con 1 unidad uculta, Recuperado de: Izenman, Alan Julian. Modern multivariate statistical techniques. Regression, classification and manifold learning (2008).

Una red neuronal de perceptrones multicapa de "alimentación hacia atrás" o red feedforward es una técnica estadística multivariada que de manera no lineal, mapea un vector de entrada $\boldsymbol{X}=\left(X_{1}, \ldots, X_{r}\right)^{T}$ de variables sobre un vector de salida $\boldsymbol{Y}=\left(Y_{1}, \ldots, Y_{s}\right)^{T}$ de variables. Entre los vectores de entrada y salida se posicionan variables "ocultas" organizadas en capas. Las variables ocultas y de salida son conocidas tradicionalmente como nodos, neuronas o unidades de procesamiento. En la figura (7) se muestra una red neuronal multicapa con dos unidades de procesamiento (una oculta y una de salida) y $r=3$ nodos de entrada, $s=2$ nodos de salida y $t=2$ nodos en la capa oculta.

La arquitectura de una red neuronal multicapa se define por sus $r$ nodos de entrada $X_{1}, \ldots, X_{r}$; uno o más capas de nodos "ocultos" y $s$ nodos de salida $Y_{1}, \ldots, Y_{s}$. Los nodos de las capas ocultas no pertenecen a las capas de entrada o salida y las unidades computacionales se encuentran en los nodos ocultos y los nodos de salida por lo que si existen $L$ capas ocultas la red es llamada "red de $(L+1)$ capas".

\subsection{Red con una capa oculta}

Teniendo una red de dos capas con $r$ nodos de entrada $\left(X_{m}, m=1,2, \ldots, r\right)$, una capa simple $(L=1)$ de $t$ nodos ocultos $\left(Z_{j}, j=1,2, \ldots, t\right)$ y $s$ nodos de salida $\left(Y_{k}, k=1,2, \ldots, s\right)$. Sea $\beta_{m j}$ el peso de la conexión $X_{m} \rightarrow Z_{j}$ con sesgo $\beta_{0 j}$ y sea $\alpha_{j k}$ el peso de la conexión $Z_{j} \rightarrow Y_{k}$ con sesgo $\alpha_{0 k}$. 
Teniendo $\boldsymbol{X}=\left(X_{1}, \ldots, X_{r}\right)^{T}$ y $\boldsymbol{Y}=\left(Y_{1}, \ldots, Y_{s}\right)^{T}$. Sea $U_{j}=\beta_{0 j}+\boldsymbol{X}^{T} \boldsymbol{\beta}_{j}$ y $V_{k}=\alpha_{0 k}+\boldsymbol{Z}^{T} \boldsymbol{\alpha}_{k}$. Entonces,

$$
\begin{gathered}
Z_{j}=f\left(U_{j}\right), j=1,2, \ldots, t, \\
\mu_{k}(\boldsymbol{X})=g_{k}\left(V_{k}\right), k=1,2, \ldots, s,
\end{gathered}
$$

donde $\beta_{j}=\left(\beta_{1 j}, \ldots, \beta_{r j}\right)^{T}$ y $\boldsymbol{\alpha}_{k}=\left(\alpha_{1, k}, \ldots, \alpha_{t, k}\right)^{T}$. Juntando todas las ecuaciones, el valor del $k$-ésimo nodo de salida puede ser expresado como

$$
Y_{k}=\mu_{k}(\boldsymbol{X})+\epsilon_{k}
$$

donde

$$
\mu_{k}(\boldsymbol{X})=g_{k}\left(\alpha_{0 k}+\sum_{j=1}^{t} \alpha_{j k} f_{j}\left(\beta_{0 j}+\sum_{m=1}^{r} \beta_{m j} X_{m}\right)\right)
$$

$k=1,2, \ldots, s, \mathrm{y}$ la $f_{j}(),. j=1,2, \ldots, t$, y la $g_{k}(),. k=1,2, \ldots, s$, son funciones de activación para cada uno de los nodos de las capas ocultas y de salida, respectivamente.

\subsection{Más de una capa oculta}

Se puede expresar la ecuación (17) en notación matricial de la siguiente manera:

$$
\boldsymbol{\mu}(\boldsymbol{X})=\mathbf{g}\left(\boldsymbol{\alpha}_{0}+A \mathbf{f}\left(\beta_{0}+B X\right)\right)
$$

Donde $\boldsymbol{B}=\left(\beta_{i j}\right)$ es una matriz $(t \mathrm{x} r)$ de pesos entre los nodos de entrada y la capa oculta, $\boldsymbol{A}=\left(\alpha_{j k}\right)$ es una matriz $(s \mathrm{x} t)$ de pesos entre la capa oculta y los nodos de salida, $\boldsymbol{\beta}_{0}=\left(\beta_{01}, \ldots, \beta_{0 t}\right)^{T}, \mathrm{y}$ $\boldsymbol{\alpha}_{0}=\left(\alpha_{01}, \ldots, \alpha_{0 s}\right)^{T}$; también $\mathbf{f}=\left(f_{1}, \ldots, f_{t}\right)^{T}$ y $\mathbf{g}=\left(g_{1}, \ldots, g_{s}\right)^{T}$ son los vectores de funciones de activación no lineales. En la ecuación (18), la notación $\mathbf{h}(\boldsymbol{U})$ representa el vector $\left(h_{1}\left(U_{1}\right), \ldots, h_{t}\left(U_{t}\right)\right)^{T}$, donde $\mathbf{h}=\left(h_{1}, \ldots, h_{t}\right)^{T}$ es un vector de funciones y $\boldsymbol{U}=\left(U_{1}, \ldots, U_{t}\right)^{T}$ es un vector aleatorio, teniendo en cuenta también que $\boldsymbol{\mu}(\boldsymbol{X})=\left(\mu_{1}(\boldsymbol{X}), \ldots, \mu_{s}(\boldsymbol{X})\right)^{T}$, lo que permite una extensión para casos en los que se tiene más de una capa oculta.

\subsection{Criterio de optimalidad}

Sea el vector $(s t+r t+t+s)$-dimensional $\boldsymbol{\omega}$ compuesto de los parámetros de una red completamente conectada (todas las capas están completamente conectadas una a la otra por sus nodos). Los pesos de conexión (elementos de las matrices $\boldsymbol{A}$ y $\boldsymbol{B}$ ) y los sesgos (los vectores $\boldsymbol{\alpha}_{0}$ y $\boldsymbol{\beta}_{0}$ ). Para estimar $\boldsymbol{\omega}$ en un caso de clasificación binaria o en problemas de regresión multivariada, es necesario minimizar la suma de errores al cuadrado (SCE):

$$
\operatorname{SCE}(\boldsymbol{\omega})=\sum_{i=1}^{n}\left\|\boldsymbol{Y}_{i}-\tilde{\boldsymbol{Y}}_{i}\right\|^{2}
$$

Con respecto a los elementos de $\omega$, se tiene 


$$
\left\|\boldsymbol{Y}_{i}-\tilde{\boldsymbol{Y}}_{i}\right\|^{2}=\left(\boldsymbol{Y}_{i}-\tilde{\boldsymbol{Y}}_{i}\right)^{T}\left(\boldsymbol{Y}_{i}-\tilde{\boldsymbol{Y}}_{i}\right)=\sum_{k \in \mathcal{K}}\left(Y_{i, k}-\tilde{Y}_{i, k}\right)^{2}
$$

y $\mathcal{K}$ es el conjunto de nodos de salida. En los problemas de clasificación binaria solo hay un nodo de salida. En la ecuación (20), $\boldsymbol{Y}_{i}=\left(Y_{i, k}\right)$ es el vector s-dimensional de valores verdaderos o valores "objetivo", $\tilde{\boldsymbol{Y}}_{i}=\left(\tilde{Y}_{i, k}\right)$ es el vector s-dimensional de valores estimados, y $\tilde{Y}_{i, k}=\mu_{k}\left(\boldsymbol{X}_{i}\right)=\mu_{k}\left(\boldsymbol{X}_{i}, \boldsymbol{\omega}\right)$ es el valor estimado en el $k$-ésimo nodo de salida correspondiente al $i$-ésimo vector $r$-dimensional de entrada $\boldsymbol{X}_{i}, k \in \mathcal{K}, i=1,2, \ldots, n$.

Para problemas de clasificación que involucran más de dos clases, usualmente se tienen $K$ nodos de salida para cada una de las $K>2$ clases posibles, uno para cada clase. En este caso, un criterio de error es menos el logaritmo de la función de verosimilitud condicional,

$$
E(\boldsymbol{\omega})=-\sum_{i=1}^{n} \sum_{k \in \mathcal{K}} Y_{i, k} \log \tilde{Y}_{i, k}, \tilde{Y}_{i, k}=\frac{e^{V_{i, k}}}{\sum_{l \in \mathcal{K}} e^{V_{i, l}}},
$$

donde $Y_{i, k}=1$ si $\boldsymbol{X}_{i} \in \prod_{k}$ y cero en otro caso, y $V_{i, k}=\alpha_{0, k}+\boldsymbol{Z}_{i}^{T} \boldsymbol{\alpha}_{k}$ es el valor de $V_{k}$ para el $i$-ésimo vector de entrada $\boldsymbol{X}_{i}$. Este criterio es equivalente al desvío de Kullback-Leibler (o entropía cruzada), y $\tilde{Y}_{i, k}$ es conocida como la función softmax, la cual es la generalización multiclase de la función logística.

Dado que el valor estimado $\tilde{Y}_{i, k}$, es una función no lineal de $\boldsymbol{\omega}$, los criterios de $S C E$ y $E$ son funciones no lineales de $\boldsymbol{\omega}$. El $\boldsymbol{\omega}$ que minimiza $S C E(\boldsymbol{\omega})$ o $E(\boldsymbol{\omega})$ no está disponible de forma explícita por lo que se recurre al algoritmo de optimización no lineal conocido como "algoritmo de retropropagación de los errores".

\subsection{Algoritmo de retropropagación de los errores}

El algoritmo de retropropagación (Werbos, 1974) calcula eficientemente las primeras derivadas de una función de error con respecto a los pesos de la red $\left\{\alpha_{k j}\right\}$ y $\left\{\beta_{j m}\right\}$. Estas derivadas son usadas para estimar los pesos, minimizando la función de error a través de un método iterativo de gradiente descendiente.

Para simplificar la descripción del algoritmo, se usa una red de una capa oculta con un nodo en ella; sin embargo, todo se puede generalizar a una red con más de un nodo oculto. Se denota a $\mathcal{M}$ como el conjunto de $m$ nodos de entrada, $\mathcal{J}$ el conjunto de $j$ nodos ocultos, y $\mathcal{K}$ el conjunto de $k$ nodos de salida, de tal forma que $m \in \mathcal{M}$ indexa un nodo de entrada, $j \in \mathcal{J}$ indexa un nodo oculto, y $k \in \mathcal{K}$ indexa un nodo de salida. En otras palabras $m \rightarrow j \rightarrow k$. Tal y como se ha venido trabajando, los vectores $r$-dimensionales de entrada son indexados por $i=1,2, \ldots, n$.

Iniciando en el $k$-ésimo nodo de salida, la señal de error en ese nodo es la siguiente:

$$
e_{i, k}=Y_{i, k}-\tilde{Y}_{i, k}, k \in \mathcal{K}
$$

y la suma de errores al cuadrado (usualmente conocida como la función de error) en cada nodo es:

$$
E_{i}=\frac{1}{2} \sum_{k \in \mathcal{K}} e_{i, k}^{2}=\frac{1}{2} \sum_{k \in \mathcal{K}}\left(Y_{i, k}-\tilde{Y}_{i, k}\right)^{2}, i=1,2, \ldots, n
$$

El criterio de optimización es la suma de errores al cuadrado (SCE) del conjunto de datos; que es, la función de error (22) promediada sobre todos los datos del conjunto de aprendizaje: 


$$
S C E=\frac{1}{n} \sum_{i=1}^{n} E_{i}=\frac{1}{2 n} \sum_{i=1}^{n} \sum_{k \in \mathcal{K}} e_{i, k}^{2} .
$$

El problema de aprendizaje es minimizar la $S C E$ a través de los pesos de conexión $\left\{\alpha_{k j}\right\}$ y $\left\{\beta_{j m}\right\}$. Dado que cada derivada de la suma de errores al cuadrado a través de los pesos de conexión es una suma sobre el set de datos de entrenamiento de las derivadas de $E_{i}, i=1,2, \ldots, n$, es posible minimizar cada $E_{i}$ por separado. La figura (8) será de utilidad para la explicación del algoritmo.

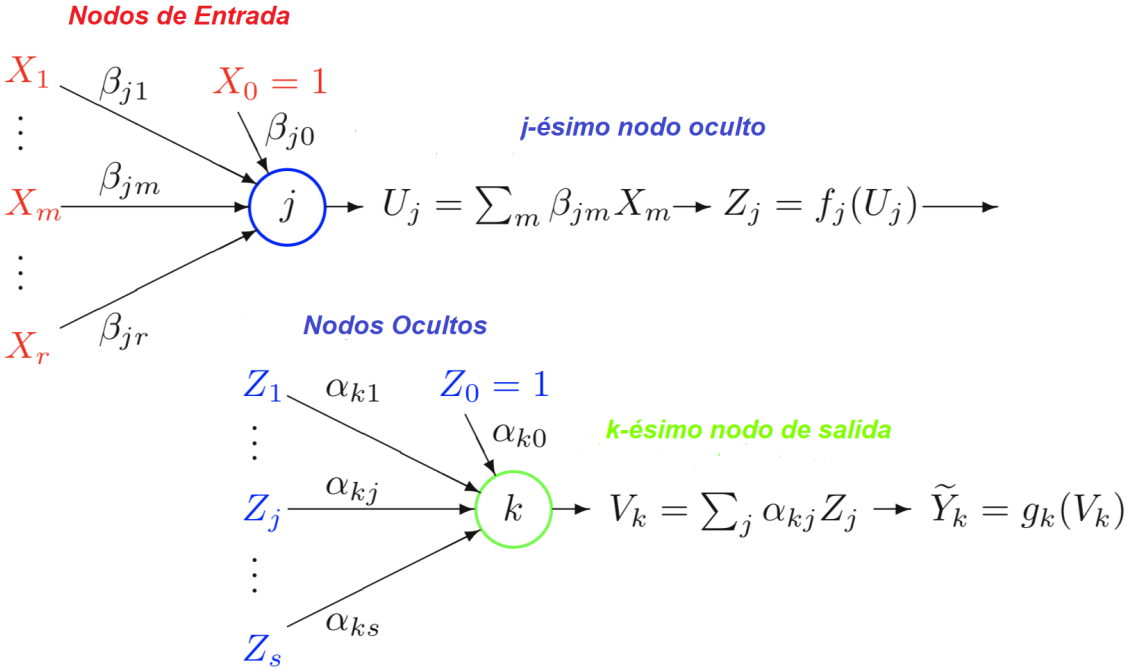

Figura 8: Esquema de algoritmo de retropropagación de los errores para una red de una capa oculta, Recuperado de: Izenman, Alan Julian. Modern multivariate statistical techniques. Regression, classification and manifold learning (2008).

Para el $i$-ésimo vector de entrada, sea

$$
V_{i, k}=\sum_{j \in \mathcal{J}} \alpha_{k j} Z_{i, j}=\alpha_{k 0}+Z_{i}^{T} \alpha_{k}, \quad k \in \mathcal{K}
$$

una suma ponderada de entradas provenientes del conjunto de unidades ocultas en el $k$-ésimo nodo de salida donde,

$$
\boldsymbol{Z}_{i}=\left(Z_{i}, 1, \ldots, Z_{i, t}\right)^{T}, \quad \boldsymbol{\alpha}_{k}=\left(\alpha_{k 1}, \ldots, \alpha_{k t}\right)^{T}
$$

y $Z_{i, 0}=1$. Entonces, la salida correspondiente es

$$
\tilde{Y}_{i, k}=g_{k}\left(V_{i}, k\right), \quad k \in \mathcal{K}
$$

donde $g_{k}($.$) es una función de activación de salida, la cual se asume diferenciable.$

El algoritmo de retropropagación es un algoritmo iterativo basado en gradientes descendientes. Usando valores iniciales generados aleatoriamente para los pesos, se busca la dirección hacia la cual la función del error decrece más rápido. 
Considere los pesos $\alpha_{i, k j}$ del $j$-ésimo nodo oculto hacia el $k$-ésimo nodo de salida. Sea $\boldsymbol{\alpha}_{i}=$ $\left(\boldsymbol{\alpha}_{i, 1}^{T}, \ldots, \boldsymbol{\alpha}_{i, s}^{T}\right)^{T}=\left(\alpha_{i, k j}\right)$ el $t$-ésimo vector de todos los pesos de conexión entre la capa oculta y la capa de salida en la $i$-ésima iteración. Entonces la regla de actualización es:

$$
\boldsymbol{\alpha}_{i+1}=\boldsymbol{\alpha}_{i}+\Delta \boldsymbol{\alpha}_{i},
$$

donde

$$
\triangle_{\alpha_{i}}=-\eta \frac{\partial \boldsymbol{E}_{\boldsymbol{i}}}{\partial \boldsymbol{\alpha}_{\boldsymbol{i}}}=\left(-\eta \frac{\partial \boldsymbol{E}_{\boldsymbol{i}}}{\partial \alpha_{i, j h}}\right)=\left(\Delta \alpha_{i, k j}\right) .
$$

Ecuaciones de actualización similares se tienen para $\alpha_{i, k 0}$. En (29), el parámetro de aprendizaje $\eta$ específica que tan amplio debe ser cada paso en el proceso iterativo. Si $\eta$ es muy grande, las iteraciónes se moverán rápidamente hacia un mínimo local y podría sobrepasarlo, pero, si $\eta$ es demasiado pequeño las iteraciónes podrían tomar un largo tiempo antes de encontrar un punto cerca a un mínimo local.

Usando la regla de la cadena para derivación, se tiene que

$$
\begin{aligned}
\frac{\partial E_{i}}{\partial \alpha_{i, k j}} & =\frac{\partial E_{i}}{\partial e_{i, k}} \cdot \frac{\partial e_{i, k}}{\partial \tilde{Y}_{i, k}} \cdot \frac{\partial \tilde{Y}_{i, k}}{\partial V_{i, k}} \cdot \frac{\partial V_{i, k}}{\partial \alpha_{i, k j}} \\
& =e_{i k} \cdot(-1) \cdot g_{k}^{\prime}\left(V_{i, k}\right) \cdot Z_{i, j} \\
& =-e_{i, k} g_{k}^{\prime}\left(\alpha_{i}, k 0+\boldsymbol{Z}_{i}^{T} \alpha_{i, k}\right) Z_{i j}
\end{aligned}
$$

Esto puede ser expresado también como

$$
\frac{\partial E_{i}}{\partial \alpha_{i, j h}}=-\delta_{i, k} Z_{i, j}
$$

donde

$$
\delta_{i, k}=-\frac{\partial E_{i}}{\partial \tilde{Y}_{i, k}} \cdot \frac{\partial \tilde{Y}_{i, k}}{\partial V_{i, k}}=e_{i, k} g_{k}^{\prime}\left(V_{i, k}\right)
$$

es la sensibilidad (o gradiente local) de la $i$-ésima observación en el $k$-ésimo nodo de salida. La expresión para $\delta_{i, k}$ es el producto de dos términos asociados con el $k$-ésimo nodo: la señal de error $e_{i, k}$ y la derivada, $g_{k}^{\prime}\left(V_{i, k}\right)$, de la función de activación. La actualización de gradiente descendiente de $\alpha_{i, k j}$ es dada por

$$
\alpha_{i+1, k j}=\alpha_{i, k j}-\eta \frac{\partial E_{i}}{\partial \_\alpha_{i, k j}}=\alpha_{i, k j}+\eta \delta_{i, k} Z_{i, j},
$$

La siguiente parte del algoritmo de retropropagación es derivar una regla de actualización para la conexión entre $m$-ésimo nodo de entrada al $j$ - ésimo nodo oculto. En la iteración $i$ se tiene,

$$
U_{i, j}=\sum_{m \in \mathcal{M}} \beta_{i, j m} X_{i, m}=\beta_{i, j 0}+\boldsymbol{X}_{i}^{T} \beta_{i, j}, \quad j \in \mathcal{J},
$$

sea la suma ponderada de entradas en el $j$-ésimo nodo, donde 


$$
\boldsymbol{X}_{i}=\left(X_{i}, 1, \ldots, X_{i, r}\right)^{T}, \quad \beta_{i, j}=\left(\beta_{i, j, 1} \ldots, \beta_{i, j r}\right)^{T},
$$

y $X_{i, 0}=1$. La salida correspondiente es

$$
Z_{i, j}=f_{j}\left(U_{i, j}\right),
$$

donde $f_{j}($.$) es la función de activación, que se asume diferenciable, en el j$-ésimo nodo oculto. Sea $\boldsymbol{\beta}_{i}=\left(\boldsymbol{\beta}_{i, 1}^{T}, \ldots, \boldsymbol{\beta}_{i, t}^{T}\right)=\left(\beta_{i, j m}\right)$ la $i$-ésima iteración del $(r+1) t$-ésimo vector de todos los pesos entre las capas de entrada a las capas ocultas. Entonces, la regla de actualización es

$$
\beta_{i+1}=\beta_{i}+\Delta \beta_{i}
$$

donde

$$
\Delta \boldsymbol{\beta}_{i}=-\eta \frac{\partial E_{i}}{\partial \boldsymbol{\beta}_{i}}=\left(-\eta \frac{\partial E_{i}}{\partial \beta_{i, j m}}\right)=\left(\Delta \beta_{i, j m}\right) .
$$

De nuevo, formulas similares de actualización pueden ser derivadas para el término de sesgo $\beta_{i, j 0}$. Usando la regla de la cadena se obtiene

$$
\frac{\partial E_{i}}{\partial \beta i, k j}=\frac{\partial E_{i}}{\partial Z_{i j}} \cdot \frac{\partial Z_{i, j}}{\partial U_{i, j}} \cdot \frac{\partial U_{i, j}}{\beta_{i, k j}} .
$$

El primer término del lado derecho de la ecuación es

$$
\begin{aligned}
\frac{\partial E_{i}}{\partial Z_{i, j}} & =\sum_{k \in \mathcal{K}} e_{i, k} \cdot \frac{\partial e_{i, k}}{\partial Z_{i, j}} \\
& =\sum_{k \in \mathcal{K}} e_{i, k} \cdot \frac{\partial e_{i, k}}{\partial V_{i, k}} \cdot \frac{\partial V_{i, k}}{\partial Z_{i, j}} \\
& =-\sum_{k \in \mathcal{K}} e_{i, k} \cdot g_{k}^{\prime}\left(V_{i, j}\right) \cdot \alpha_{i, k j} \\
& =-\sum_{k \in \mathcal{K}} \delta_{i, k} \alpha_{i, k j},
\end{aligned}
$$

por lo tanto, de la ecuación (39) se obtiene,

$$
\frac{\partial E_{i}}{\partial \beta_{i, k j}}=-\sum_{k \in \mathcal{K}} e_{i, k} g_{k}^{\prime}\left(\alpha_{i, k 0}+\boldsymbol{Z}_{i}^{T} \boldsymbol{\alpha}_{i, k}\right) \alpha_{i, k j} f_{j}^{\prime}\left(\beta_{i, j 0}+\boldsymbol{X}_{i}^{T} \boldsymbol{\beta}_{i, j}\right) X_{i, m}
$$

Juntando las ecuaciones (32) y (40), se logra

$$
\delta_{i, j}=f_{j}^{\prime}\left(U_{i, j}\right) \sum_{k \in \mathcal{K}} \delta_{i, j} \alpha_{i, j k} .
$$

Esta expresión para $\delta_{i, j}$ es el producto de dos términos: el primero $f_{j}^{\prime}\left(U_{i, j}\right)$, es la derivada de la función de activación $f_{j}($.) evaluada en el $j$-ésimo nodo oculto; el segundo término es una suma 
ponderada de todos los $\delta_{i, k}$ (que requieren el conocimiento del error $e_{i, k}$ en el $k$-ésimo nodo de salida) sobre todos los nodos de salida, donde el $k$-ésimo peso, $\alpha_{i, k j}$, es el peso de conexión del $j$-ésimo nodo oculto con el $k$-ésimo nodo de salida.

La actualización de gradiente descendiente de $\beta_{i, j m}$ está dada por

$$
\beta_{i+1, j m}=\beta_{i+1, j m}-\eta \frac{\partial E_{i}}{\partial \beta_{i, j m}}=\beta_{i, j m}+\eta \delta_{i, j} X_{i, m},
$$

donde $\eta$ es el parámetro de tasa de aprendizaje del algoritmo de retropropagación.

El algoritmo esta entonces definido por las ecuaciones (32) y (43). Dichas fórmulas de actualización indican la existencia de dos etapas en el algoritmo: una etapa de "paso de retroalimentación" y otra de "paso de retropropagación". Luego de un paso de inicialización en el cual los pesos son asignados de manera aleatoria, se tienen las siguientes etapas:

- Paso de Retroalimentación: Las entradas ingresan al nodo de la izquierda y este calcula una salida como en las ecuaciones (25) y (26). Los resultados son pasados de derecha a izquierda a través de las capas de la red.

- Paso de Retropropagación: La red funciona en orden inverso, de derecha a izquierda, empezando en la capa de salidas.

- El error de la ecuación (22) es calculado en el $k$-ésimo nodo de salida y luego es multiplicado por la derivada de la función de activación para obtener la sensitividad $\delta_{i . k}$ en el nodo de salida según la expresión (32); los pesos, $\left\{\alpha_{i, k j}\right\}$ que alimentan los nodos de salida son actualizados por la expresión (33).

- Se usa la ecuación (42) para calcular la sensibilidad $\delta_{i, j}$ en el $j$-ésimo nodo oculto, y luego, se usa la expresión (43) para actualizar los pesos, $\left\{\beta_{i, j m}\right\}$, alimentando los nodos ocultos.

El proceso se repite de manera iterativa hasta algún tiempo o criterio de parada definido.

\section{Arboles de clasificación.}

El algoritmo que fundamenta los árboles de regresión o clasificación (CART por sus siglas en inglés) se denomina "algoritmo de partición recursiva". El algoritmo construye el árbol de decisión mediante la partición de las hojas en más nodos. La partición de cada hoja se realiza seleccionando un valor de una variable o característica de los individuos que genere la mejor partición (secuencia de preguntas jerárquicas boleanas), permitiendo la predicción de una clase (Breiman, Friedman, Olshen \& Stone, 1984).

El punto de inicio de un árbol de clasificación se denomina nodo raíz y se compone de todo el conjunto de aprendizaje $\mathcal{L}$ posicionado en la parte superior del árbol. Un nodo es un subconjunto del conjunto de variables y puede ser un nodo terminal o no terminal, y su división binaria está determinada por una condición booleana determinada por el valor de una sola variable, donde la condición se cumple ("sí") o no se cumple ("no") por el valor observado de esa variable. Cuando un nodo no se divide, es llamado denomina nodo terminal y se le asigna una etiqueta de clase, pero cuando posee una división de otra variable se denomina nodo no terminal. 


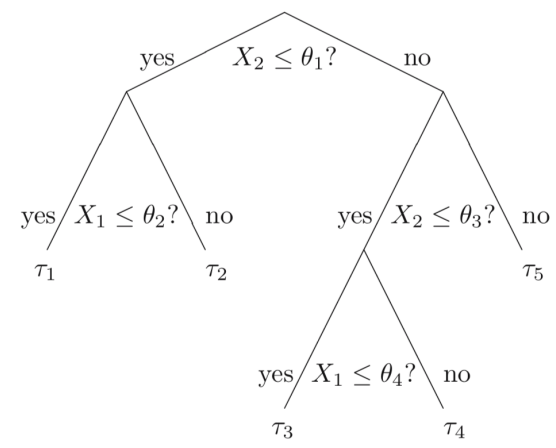

Figura 9: Diagrama de partición recursiva para dos variables, Recuperado de: Izenman, Alan Julian. Modern multivariate statistical techniques. Regression, classification and manifold learning (2008).

Un ejemplo de partición recursiva mediante árboles se presenta en la figura (9). En ella se tienen dos variables $X_{1}$ y $X_{2}$ y la partición se realiza en tres etapas, y el proceso (desde el primer al segundo nodo) es:

Primero se realiza la pregunta ¿es $X_{2} \leq \theta_{1}$ ?, si la respuesta es $s i$; se prosigue a la rama izquierda, de lo contrario se sigue por la rama derecha. Segundo, si la respuesta en la etapa uno es si, se realiza la siguiente pregunta ¿es $X_{1} \leq \theta_{2}$ ? Una respuesta de si representa el nodo terminal $\tau_{1}$ con la correspondiente región $R_{1}=\left\{X_{1} \leq \theta_{2}, X_{2} \leq \theta_{1}\right\}$; mientras que una respuesta de no implica finalizar en el nodo terminal $\tau_{2}$ con la región correspondiente $R_{2}=\left\{X_{1}>\theta_{2}, X_{2} \leq \theta_{1}\right\}$. El proceso sigue iterativamente hasta obtener las regiones $R_{3}, R_{4}$ y $R_{5}$ correspondientes a los nodos terminales $\tau_{3}, \tau_{4}$ y $\tau_{5}$.

\subsection{Estrategias de división:}

Para hacer crecer un árbol de clasificación, es necesario responder cuatro preguntas básicas: cómo escoger las condiciones booleanas que van a dividir los nodos, que criterio usar para dividir un nodo padre en dos nodos hijos, cuando establecer que un nodo es un nodo terminal y como asignar una clase a un nodo terminal.

En cada nodo, el algoritmo encargado de hacer crecer el árbol debe tener la capacidad de encontrar cual variable es la "mejor" para hacer la partición. Para hacerlo, se deben evaluar todas las variables e identificar todas las posibles particiones.

Se debe tener en cuenta que se debe hacer una distinción entre variables ordinales (o continuas) y nominales (o categóricas).

\subsubsection{División de una variable categórica}

Teniendo una variable categórica con $M$ categorías distintas, $l_{1}, \ldots, l_{M}$, el conjunto $\mathcal{S}$ de posibles divisiones de un nodo para la variable está conformado el conjunto de todos los subconjuntos de $\left\{l_{1}, \ldots, l_{M}\right\}$. Denotando a $\tau_{I}$ y $\tau_{D}$ como los nodos hijo izquierdo e hijo derecho respectivamente, provenientes de un nodo padre $\tau$, si se tienen $M=4$ categorías, entonces hay $2^{M}-2=14$ posibles divisiones (ignorando las divisiones donde uno de los nodos hijos está vacío). Entonces, el conjunto $\mathcal{S}$ de siete divisiones distintas viene dado por la siguiente tabla: 
Cuadro 2: Posibles divisiones de nodo para una variable categórica

\begin{tabular}{cc}
\hline$\tau_{I}$ & $\tau_{D}$ \\
\hline$l_{1}$ & $l_{2}, l_{3}, l_{4}$ \\
$l_{2}$ & $l_{1}, l_{3}, l_{4}$ \\
$l_{3}$ & $l_{1}, l_{2}, l_{4}$ \\
$l_{4}$ & $l_{1}, l_{2}, l_{3}$ \\
$l_{1}, l_{2}$ & $l_{3}, l_{4}$ \\
$l_{1}, l_{3}$ & $l_{2}, l_{4}$ \\
$l_{1}, l_{4}$ & $l_{2}, l_{3}$
\end{tabular}

Recuperado de: Izenman, Alan Julian. Modern multivariate statistical techniques. Regression, classification and manifold learning (2008).

\subsubsection{Funciones de impureza del nodo.}

Para elegir la mejor división entre todas las variables, primero se debe elegir la mejor división para una variable en específico. Teniendo $\prod_{1}, \ldots, \prod_{K}$ las $K>2$ clases, para el nodo $\tau$, se define la función de impureza del nodo $i(\tau)$ como

$$
i(\tau)=\phi(p(1 \mid \tau), \ldots p(K \mid \tau))
$$

donde $p(k \mid \tau)$ es una estimación de $P\left(\boldsymbol{X} \in \prod_{k} \mid \tau\right)$, la probabilidad condicional de que una observación $\boldsymbol{X}$ pertenezca a la clase $\prod_{k}$ dado que cae en el nodo $\tau$. En la ecuación 44 es necesario que $\phi$ sea una función simétrica definida en el conjunto de todos los $k$-tuplas de probabilidad $\left(p_{1}, \ldots, p_{k}\right)$, minimizadas en los puntos $(1,0, \ldots, 0),(0,1,0, \ldots, 0), \ldots(0,0, \ldots, 0,1)$ y se maximiza en los puntos $\frac{1}{K}, \ldots, \frac{1}{K}$. En el caso de dos clases $(k=2)$, esta condiciones se reducen a una función $\phi(p)$ simétrica que se maximiza en el punto $p=\frac{1}{2}$ con $\phi(0)=\phi(1)=0$.

Una de esas funciones $\phi$ es la función de entropía,

$$
i(\tau)=-\sum_{k=1}^{k} p(k \mid \tau) \log p(k \mid \tau)
$$

la cual; cuando solo hay dos clases se reduce a

$$
i(\tau)=-p \log p-(1-p) \log (1-p)
$$

cuando se establece a $p=p(1 \mid \tau)$. Otras funciones $\phi$ han sido sugeridas para medir la impureza del nodo, entre ellas, el índice de Gini definido como

$$
i(\tau)=-\sum_{k \neq k^{\prime}} p(k \mid \tau) p\left(k^{\prime} \mid \tau\right)=1-\sum_{k}\left\{p(k \mid \tau)^{2}\right\}
$$

que para el caso de $K=2$ clases se reduce a 


$$
i(\tau)=2 p(1-p)
$$

\subsubsection{Elegir la mejor división para una variable}

Suponga que, en el nodo $\tau$, se aplica la división $s$ de tal manera que una proporción $p_{L}$ de las observaciones se aloje en el nodo secundario izquierdo $\tau_{I}$ y la proporción restante $p_{R}$ se encuentre en el nodo secundario derecho $\tau_{D}$.

Iniciando en el nodo padre $\tau$ y usando la función de entropía de la ecuación (45) como medida de impureza, se estima $p_{L}$ por $n_{+1} / n_{++}$y $p R$ por $n_{+2} / n_{++}$, obteniendo la siguiente función de impureza estimada:

$$
i(\tau)=-\left(\frac{n+1}{n_{++}}\right) \log _{e}\left(\frac{n+1}{n_{++}}\right)-\left(\frac{n+2}{n_{++}}\right) \log _{e}\left(\frac{n+2}{n_{++}}\right)
$$

en la cual $i(\tau)$ es completamente independiente del tipo de división propuesta. Ahora, para los nodos hijos $\tau_{l}$ y $\tau_{R}$; y para $X_{j} \leq c$, se estima $p_{l}$ por $n_{11} / n_{1}$ y $p_{R}$ por $n_{12} / n_{1+}$, y para $X_{j}>c$ se estima $P L$ para $n_{21} / n_{2}+$ y $p_{R}$ por $n 22 / n_{2}+$, sobre lo que se calcula

$$
\begin{aligned}
& i(\tau)=-\left(\frac{n_{11}}{n_{1+}}\right) \log _{e}\left(\frac{n_{11}}{n_{1+}}\right)-\left(\frac{n_{12}}{n_{1+}}\right) \log _{e}\left(\frac{n_{12}}{n_{1+}}\right) \\
& i(\tau)=-\left(\frac{n_{21}}{n_{2+}}\right) \log _{e}\left(\frac{n_{11}}{n_{1+}}\right)-\left(\frac{n_{22}}{n_{2+}}\right) \log _{e}\left(\frac{n_{22}}{n_{2+}}\right)
\end{aligned}
$$

La bondad de la división $s$ en el nodo $\tau$ viene dada por la reducción de la impureza obtenida al dividir el nodo padre $\tau$ en sus nodos hijos, $\tau_{R}$ y $\tau_{L}$

$$
\triangle_{i}(s, \tau)=i(\tau)-p_{L} i\left(\tau_{L}\right)-p_{R} i\left(\tau_{R}\right)
$$

La mejor división para la variable $X_{j}$ es la que tiene mayor valor de $\triangle_{i}(s, \tau)$ sobre todas las $s \in S_{j}$, con $S_{j}$ como el conjunto de posibles divisiones distintas para $X_{j}$.

\section{Modelos ensamblados}

Controlar la inestabilidad de un predictor (sesgo versus varianza) es una tarea crucial en los algoritmos de aprendizaje de máquina, la cual depende del set de datos usado para entrenar el modelo (cuando un pequeño cambio en los datos induce un cambio drástico en el predictor, este es inestable). Los predictores basados en redes neuronales y árboles de clasificación son generalmente inestables, pues tienen una alta varianza y sesgos muy bajos (altas tasas de precisión).

Sin embargo, la inestabilidad de un modelo puede ser usada como parámetro para mejorar la precisión del algoritmo. Las metodologías de "bagging" y "boosting" explotan la presencia de inestabilidad en un predictor para crear un método de aprendizaje más certero, creando perturbaciones en el set de datos y creando ensamblajes de diferentes predictores que al ser combinados se convierten en un único modelo. 


\subsection{Bagging}

Ensamblar modelos fue el primer procedimiento que combinó con éxito un conjunto de algoritmos de aprendizaje para mejorar el rendimiento en un solo algoritmo de este tipo. Los modelos ensamblados son mas exitosos si el predictor es inestable. Si el procedimiento de aprendizaje es estable, los modelos ensamblados no diferirán mucho del predictor único e incluso puede debilitar su rendimiento. Sin embargo, cuando el procedimiento de aprendizaje es inestable, se tiende a ver una mejora significativa para el predictor del modelo con respecto al procedimiento inestable original (Izenman, 2008).

Por lo tanto se puede denotar el conjunto de aprendizaje de $n$ observaciones por

$$
\mathcal{L}=\left\{\left(\boldsymbol{X}_{i}, Y_{i}\right), i=1,2, \ldots, n\right\}
$$

Donde $\left\{Y_{i}\right\}$ son respuestas continuas (un problema de regresión) o etiquetas de clase desordenadas (un problema de clasificación). El método toma un conjunto ensamblado de datos de entrenamiento $\left\{\mathcal{L}_{K}\right\}$ (el mismo conjunto para cada modelo) con $n$ observaciones, y combina los predictores de tal manera que el predictor resultante mejore la predicción obtenida sobre un solo set de datos de entrenamiento $\mathcal{L}$

Los procedimientos para los modelos ensamblados comienzan con la generación de $B$ muestras aleatorias simples con reemplazamiento del conjunto $\mathcal{L}$. La muestras pueden ser denotadas como

$$
\mathcal{L}^{* b}=\left\{\left(\boldsymbol{X}_{i}^{* b}, Y_{i}^{* b}\right), i=1,2 \ldots, n\right\}, \quad b=1,2, \ldots, B
$$

\subsubsection{Modelos ensamblados basados en arboles de clasificación}

En el caso de árboles de clasificación, $Y_{i} \in\{1,2, \ldots, K\}$ es una etiqueta de clase adjunta a $\boldsymbol{X}_{i}$. Se hace crecer el árbol de clasificación $T^{* b}$ para la $b$-ésima muestra de $\mathcal{L}^{* b}$, y para reducir el sesgo se hace crecer el árbol tanto como se pueda sin podarlo ("prunning").

Suponga que $(\boldsymbol{X}, Y)$ es muestreada aleatoriamente de la misma distribución conjunta de los miembros de $\mathcal{L}$, se distribuye $\boldsymbol{X}$ en cada uno de los $B$ árboles muestreados. Para cada árbol cuando $\boldsymbol{X}$ se encuentra dentro de un nodo terminal asociado con una clase en particular se asume que el árbol "voto" por esa clase. Siguiendo esta lógica se predice la clase de $\boldsymbol{X}$ como la clase que recibió el número mayor de votos en todos los $B$ árboles (regla de mayoría de voto).

Debido a que se hace muestreo con reemplazamiento sobre $\mathcal{L}$ existe un porcentaje de observaciones que no serán seleccionadas en la $b$-ésima muestra, este conjunto de observaciones denotadas como $(\boldsymbol{X}, Y) \notin \mathcal{L}^{* b}$ son llamadas observaciones "fuera de la bolsa"(OOB por sus siglas en ingles) y pueden ser utilizadas para evaluar el ensamblaje de arboles.

Suponga $\left(\boldsymbol{X}_{i}, Y_{i}\right) \notin \mathcal{L}^{* b}$, se predice la etiqueta de clase de $\boldsymbol{X}_{i}$ con el árbol de clasificación $T^{* b}$ entrenado a partir de la muestra $\mathcal{L}^{* b}$. Teniendo $n_{i}(\leq B)$ árboles en los cuales $\boldsymbol{X}_{i}$ es un miembro de la muestra OOB correspondiente, se agregan cada uno de los votos en cada uno de los $n_{i}$ árboles para cada una de las $K$-clases obteniendo el vector $K$-dimensional

$$
\hat{p}\left(x_{i}\right)=\left(\hat{p_{1}}\left(x_{i}\right), \hat{p}_{2}\left(x_{i}\right), \ldots, \hat{p} k\left(x_{i}\right)\right)^{\tau},
$$

donde $\hat{p} k\left(x_{i}\right)$ es la proporción de los $n_{i}$ árboles que votaron para que $\boldsymbol{X}_{i}=x_{i}$ fuera un miembro de la $k$-ésima clase $\prod_{k}$. La proporción $\hat{p}_{k}\left(x_{i}\right)$ es una estimación de la probabilidad $p\left(\prod_{k} \mid x_{i}\right)=$ $P\left(\boldsymbol{X} \in \prod_{k} \mid \boldsymbol{X}=x_{i}\right)$. 


\subsection{Boosting}

El objetivo de este método de ensamblaje es el de mejorar la precisión de un algoritmo débil de clasificación binaria (aquel que logra una precisión ligeramente por encima del $50 \%$ ), creando un clasificador fuerte dándole "impulso" (boost) a un clasificador débil.

El algoritmo de boosting, combina $M$ clasificadores base $C_{1}, C_{2}, \ldots, C_{M}$ de la siguiente manera. Para una observación $\boldsymbol{X}=\boldsymbol{x}$, el clasificador potenciado está definido por:

$$
C_{\boldsymbol{\alpha}}(\boldsymbol{x})=\operatorname{sign}\left\{f_{\boldsymbol{\alpha}}(\boldsymbol{x})\right\}
$$

donde

$$
f_{\boldsymbol{\alpha}}(\boldsymbol{x})=\sum_{j=1}^{M}\left(\frac{\alpha_{j}}{\sum_{j^{\prime}} \alpha_{j^{\prime}}}\right) C_{j}(\boldsymbol{x}),
$$

y $\boldsymbol{\alpha}=\left(\alpha_{1}, \ldots, \alpha_{M}\right)^{T}$ es un vector $M$-dimensional de coeficientes constantes. Estos coeficientes, deben ser asignados de tal manera que generen el mayor aumento posible en la precisión de los clasificadores débiles; uno de los algoritmos más conocidos para esta tarea es el algoritmo AdaBoost planteado por (Freund \& Schapire, 1997).

\subsection{Penalización de parámetros}

Otra manera de evitar estimaciones sub óptimas ("overfitting") es la penalización de los parámetros estimados de un predictor. La manera más conocida de hacerlo es mediante la selección de variables que aporten más información al modelo. Sin embargo, no siempre es posible eliminar variables debido al problema de clasificación al que se este afrontando, por lo que surge otra metodología que mediante la reducción o penalización de los parámetros que no aportan mucha información, restringe su efecto sobre la predicción final.

Los predictores penalizados son obtenidos usando una función de penalización $p(\boldsymbol{\alpha})$ que restringe la magnitud del vector de coeficientes $\boldsymbol{\alpha}=\left(\alpha_{1}, \ldots, \alpha_{M}\right)^{T}$ para el caso de un predictor ensamblado (ecuación 56), sin embargo, la metodología de penalización puede ser aplicada a la mayoría de predictores, y sigue la misma lógica planteada (como por ejemplo a los pesos de conexión de una red neuronal).

Los coeficientes estimados penalizados son obtenidos mediante la solución al siguiente problema de minimización restringida:

$$
\hat{\boldsymbol{\alpha}}_{\lambda}=\underset{\alpha}{\operatorname{argmin}}\left\{\sum_{i=1}^{n} L\left(y_{i}, f\left(\boldsymbol{x}_{i}\right)\right)+\lambda p(\boldsymbol{\alpha})\right\}
$$

donde $L$ es una función de pérdida y $\lambda>0$ es un parámetro de penalización. Existen dos tipos de penalización para ser usadas tanto en el contexto de un predictor simple o en el caso de ensamblaje de modelos. La primera, conocida como penalización $\boldsymbol{L}_{1}$ los coeficientes son restringidos de tal forma que la suma de sus valores absolutos debe ser menor a un valor de tolerancia mínimo,

$$
p_{1}(\boldsymbol{\alpha})=\sum_{j=1}^{M}\left|\alpha_{j}\right|
$$


y la segunda es llamada penalización $\boldsymbol{L}_{1}$, la cual restringe la suma de cuadrados de los parámetros a un valor de tolerancia mínimo,

$$
p_{2}(\boldsymbol{\alpha})=\sum_{j=1}^{M}\left(\alpha_{j}\right)^{2}
$$

\section{Random Forest}

Los modelos de "Bosques Aleatorios" o Random Forest (RF) son una extensión de la idea de ensamblar modelos para mejorar la capacidad predicción de un modelo (Breiman, 2001). En RF, se inicia de la misma manera en que se inicia un modelo ensamblado, con $B$ muestras aleatorias simples con reemplazo, tomadas del set de entrenamiento $\mathcal{L}$, pero la diferencia radica en como se hacen crecer los árboles a partir de dichas muestras.

Se introduce un componente de aleatorización en la construcción de los $B$ árboles, de tal manera que; para el árbol $T^{* b}$, cada nodo es particionado usando un subconjunto de las variables de entrada, seleccionado aleatoriamente.

Es por lo anterior, que un modelo RF solo tiene dos parámetros de mejora ("tunning"), el número $m$ de variables a ser seleccionadas aleatoriamente del set de entrenamiento, y el número $B$ de muestras aleatorias del observaciones del set de entrenamiento. El algoritmo usado para entrenar un modelo RF se muestra a continuación:

\section{Algoritmo de clasificación Random Forest usando selección aleatoria de variables de entrada}

1. Se ingresa $\mathcal{L}=\left\{\left(\boldsymbol{x}_{i}, y_{i}\right), i=1, \ldots, n\right\}, y_{i} \in\{1,2, \ldots, K\}, m=$ número de variables a elegir en cada nodo $(m<<r)$ y $B=$ número de muestras aleatorias simples con reemplazo.

2. para $b=1, \ldots, B$ repetir:

- Seleccionar una muestra aleatoria $\mathcal{L}^{* b}$ del set de entrenamiento $\mathcal{L}$.

- A partir de $\mathcal{L}^{* b}$, hacer crecer un árbol de clasificación $T^{* b}$, seleccionando aleatoriamente $m$ de las $r$ variables, determinando la mejor división con el coeficiente de Gini y haciendo crecer el árbol hasta la máxima profundidad sin podarlo.

- El árbol $T^{* b}$, genera un vector aleatorio asociado $\boldsymbol{\theta}_{b}$, independiente de los previos $\boldsymbol{\theta}_{1}, \ldots, \boldsymbol{\theta}_{b-1}$.

- Usando $\boldsymbol{\theta}_{b}$ y un vector de entrada $\boldsymbol{x}$, se define un clasificador $h\left(\boldsymbol{x}, \boldsymbol{\theta}_{b}\right)$

3. Los $B$ clasificadores aleatorizados $\left\{h\left(\boldsymbol{x}, \boldsymbol{\theta}_{b}\right)\right\}$ conforman el "bosque aleatorio"(RF).

4. La clase de la observación $x$ es asignada mediante la regla de mayoría de votos, determinados por los árboles que conforman el bosque.

Recuperado de: Izenman, Alan Julian. Modern multivariate statistical techniques. Regression, classification and manifold learning (2008). 


\section{Extreme Gradient Boosting (XGBoost)}

La derivación de los algoritmos de impulso de árboles ("tree boosting algorithms") sigue la misma idea en la literatura existente sobre gradientes de impulso, especialmente en los métodos de segundo orden originados por (Friedman et al, 2000).

\subsection{Objetivos de aprendizaje penalizados.}

Para un conjunto de datos dado con $n$ ejemplos y $m$ características o variables $D=\left\{\left(\boldsymbol{x}_{\boldsymbol{i}}, y_{i}\right)\right\}(|D|=$ $n, \boldsymbol{x}_{\boldsymbol{i}} \in \Re^{m}, y_{i} \in \Re$ ), un modelo de ensamblado de arboles usa $K$ funciones aditivas (ecuación 61 ) para predecir la variable de interés (salida).

$$
\hat{y}_{i}=\phi\left(\boldsymbol{x}_{\boldsymbol{i}}\right)=\sum_{k=1}^{k} f_{k}\left(\boldsymbol{x}_{\boldsymbol{i}}\right), f_{k} \in f,
$$

donde $f=f(x)=w_{q(x)}\left(q: \Re^{m} \rightarrow T, w \in \Re^{T}\right)$ es el espacio de árboles de regresión (también conocido como CART). En este espacio $q$ representa la estructura de cada árbol que mapea un ejemplo para cada correspondiente indice de hoja. $T$ es el número de hojas en cada árbol y, cada $f_{k}$ corresponde a una estructura de árbol independiente $q$ y con sus respectivos pesos o ponderaciones por hoja $w$. A diferencia de los arboles de decisión, cada árbol de regresión contiene una puntuación $w_{i}$, continua en cada una de las $i$ hojas. Para un problema dado, se usan las reglas de decisión de cada árbol (dadas por $q$ ) para clasificarlas dentro de las hojas y calcular la predicción final sumando el puntaje en las hojas correspondientes (imagen 10).

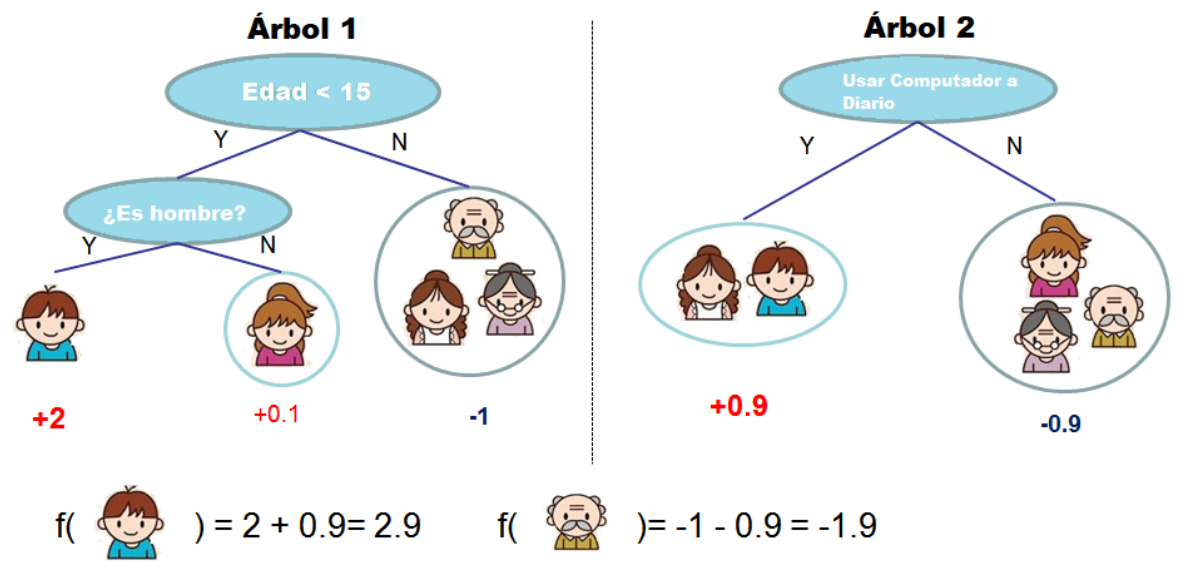

Figura 10: Modelo de Arbol Ensamblado, Recuperado de: Chen, Tianqi, and Carlos Guestrin. Xgboost: A scalable tree boosting system. Proceedings of the 22nd acm sigkdd international conference on knowledge discovery and data mining. ACM, 2016.

Para el aprendizaje o entrenamiento del modelo, dado el conjunto de funciones del modelo; el objetivo es minimizar el objetivo penalizado de la ecuación (62).

$$
\mathcal{L}(\phi)=\sum_{i} l\left(\hat{y_{i}}, y_{i}\right)+\sum_{k} \Omega\left(f_{k}\right)
$$


Donde,

$$
\Omega(f)=\gamma T+\frac{1}{2} \lambda\|\omega\|^{2}
$$

En la ecuación (62) l es una función de pérdida convexa diferenciable, que mide la distancia entre la predicción $\hat{y}_{i}$ y el objetivo $y_{i}$. El segundo término $\Omega$ se encarga de penalizar la complejidad del modelo (la función de regresión de los arboles). El término de penalización adicional ayuda a suavizar los pesos estimados para un sobre ajuste excesivo ("over-fitting"), técnica similar de penalización usada en en los modelos de árboles penalizados "avaros" ("Regularized Greedy Forest" o RGF por sus siglas en inglés). La penalización es importante, dado que si el término de penalización es igual a uno, el modelo es simplemente el resultado del algoritmo de impulso de árboles mediante gradiente.

\subsection{Gradient tree boosting}

El modelo de árboles ensamblados de la ecuación (62) usa como parámetros funciones que no se pueden optimizar mediante métodos tradicionales de optimización en el espacio euclidiano, y para lograr entrenarlo, se hace de manera aditiva. Teniendo a $\hat{y}_{i}{ }^{(t)}$ como la predicción de la $i$-ésima observación en la $t$-ésima iteración, es necesario agregar $f_{t}$ para minimizar la siguiente función objetivo.

$$
\mathcal{L}^{(t)}=\sum_{i=1}^{n} l\left(y_{i}, \hat{y}_{i}^{(t-1)}+f_{t}\left(\boldsymbol{x}_{\boldsymbol{i}}\right)\right)+\Omega\left(f_{t}\right)
$$

La ecuación (63) permite añadir el $f_{t}$ que genera una mayor mejora para el modelo de la ecuación 62. Proceso que se puede lograr usando la aproximación de segundo orden de la ecuación (64), la cual permite optimizar el objetivo de la configuración general del modelo.

$$
\mathcal{L}^{(t)} \simeq \sum_{i=1}^{n}\left[l\left(y_{i}, \hat{y}^{(t-1)}\right)+g_{i} f_{t}\left(\boldsymbol{x}_{\boldsymbol{i}}\right)+\frac{1}{2} h_{i} f_{t}^{t}\left(\boldsymbol{x}_{\boldsymbol{i}}\right)\right]+\Omega\left(f_{t}\right)
$$

En la ecuación (64) $g_{i}=\mathcal{O}_{y^{(t-1)}} l\left(y_{i}, \hat{y}^{(t-1)}\right)$ y $h_{i}=\mathcal{O}_{\hat{y}^{(t-1)}}^{2} l\left(y_{i}, \hat{y}\right)^{(t-1)}$ son las estadísticas de gradiente de primer y segundo orden de la función de pérdida, y los términos constantes pueden ser removidos para obtener una ecuación objetivo simplificada para el paso $t$ (ecuación 65)

$$
\tilde{\mathcal{L}}^{(t)}=\left[g_{i} f_{t}\left(\boldsymbol{x}_{\boldsymbol{i}}\right)+\frac{1}{2} h_{i} f_{t}^{2}\left(\boldsymbol{x}_{\boldsymbol{i}}\right)\right]+\Omega\left(f_{t}\right)
$$




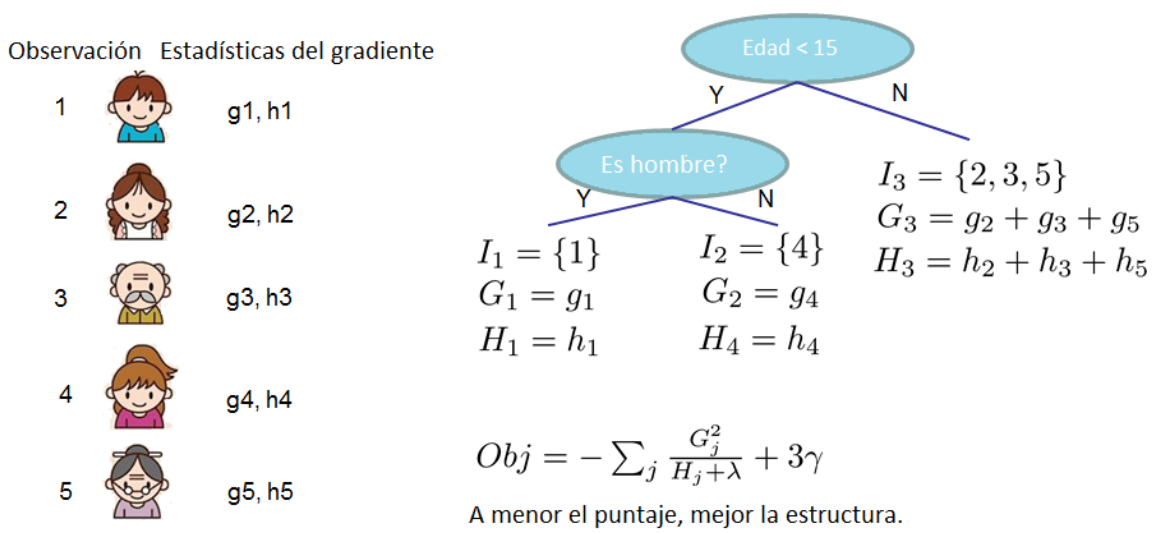

Figura 11: Estructura de Cálculo del Puntaje, Recuperado de: Chen, Tianqi, and Carlos Guestrin. Xgboost: A scalable tree boosting system. Proceedings of the 22nd acm sigkdd international conference on knowledge discovery and data mining. ACM, 2016.

Definiendo a $I_{j}=\left\{i \mid q\left(\left(\boldsymbol{x}_{\boldsymbol{i}}\right)=j\right\}\right.$ como el conjunto de observaciones de la hoja $j$, se puede re-escribir la ecuación 63 expandiendo $\Omega$ de la siguiente manera

$$
\tilde{\mathcal{L}}^{(t)}=\sum_{i=1}^{n}\left[g_{i} f_{t}\left(x_{i}\right)+\frac{1}{2} h_{i} f_{t}^{2}\left(\boldsymbol{x}_{\boldsymbol{i}}\right)\right]+\gamma T+\frac{1}{2} \lambda \sum_{j=1}^{T} w_{j}^{2}=\sum_{j=1}^{T}\left[\left(\sum_{i \in I_{j}} g_{i}\right) w_{j}+\frac{1}{2}\left(\sum_{i \in i_{j}} h_{i}+\lambda\right) w_{j}^{2}\right]+\gamma T
$$

Para una estructura fija $q(x)$, se puede calcular el peso óptimo $w_{j}^{*}$ de la hoja $j$ por:

$$
w_{j}^{*}=-\frac{\sum_{i \in I j} g_{i}}{\sum_{i \in I_{j}} h_{i}+\lambda},
$$

y se calcula el valor optimo correspondiente por:

$$
\tilde{\mathcal{L}}^{(t)}(q)=-\frac{1}{2} \sum_{j=1}^{T} \frac{\left(\sum_{i} \in I_{j} g_{i}\right)^{2}}{\sum_{i} \in I_{j} h_{i}+\lambda}+\gamma T
$$

En la ecuación (68) se puede utilizar una función de puntuación para medir la calidad de la estructura del árbol $q$. Este puntaje se puede interpretar como el puntaje de impureza usado para evaluar árboles de decisión, excepto que puede ser usado para una gama más amplia de funciones objetivo (proceso ilustrado en la figura 11).

Generalmente es imposible enumerar todos las $q$ posibles estructuras de árboles. En su lugar, se utiliza un algoritmo "ávaro" o "Greedy Algorithm" que comienza a partir de una sola hoja y agrega de manera iterativa más ramas al árbol. Asumiendo que $I_{L}$ y $I_{R}$ son los conjuntos de observaciones de nodos izquierdo y derecho después de la división, y definiendo $I=I_{L} \cup I_{R}$; entonces la reducción de la pérdida después de la división está dada por (69)

$$
\mathcal{L}_{\text {split }}=\frac{1}{2}\left[\frac{\left(\sum_{i \in I_{L}} g_{i}\right)^{2}}{\sum_{i \in I_{l}} h_{i}+\lambda}+\frac{\left(\sum_{i \in I_{R}} g_{i}\right)^{2}}{\sum_{i \in I_{R}} h_{i}+\lambda}-\frac{\left(\sum_{i \in I} g_{i}\right)^{2}}{\sum_{i \in I} h_{i}+\lambda}\right]-\gamma
$$

Fórmula usada comúnmente para evaluar los candidatos de separación. 


\section{Datos Disponibles.}

\subsection{StockTwits}

SockTwits es una plataforma de comunicación tipo red social, diseñada para traders e inversionistas; para discutir mediante textos cortos sobre stocks y el mercado. Los usuarios, al igual que un hashtag en twitter (\#hashag); tienen la posibilidad de etiquetar el activo sobre el que están hablando mediante el "cashtag", o el símbolo de dolar seguido de la abreviatura del activo usada en la bolsa (ej \$AAPL para Apple Inc.). Esta metodología permite que la minería de datos y el análisis de datos sobre los usuarios y los stocks sea más efectiva.

\subsubsection{StockTwits API}

Como se mencionó en el apartado anterior StockTwits es una red social para traders, inversionistas y entusiastas del tema de la bolsa y el mercado de títulos valores. Mediante esta comparten ideas y generan información masiva sobre el mercado en tiempo real. Una gran parte de esta información está disponible al público mediante la API de desarrollador que provee la plataforma. A través de esta se pueden obtener hasta 30 paquetes de datos acerca de un \$TICKER por hora. Esta API permite a los usuarios obtener información sin necesidad de registro (Limitada) acerca de la actividad de los usuario y el mercado, o ya más específica relacionada con permisos de inicio de sesión creación de usuarios y descarga menos limitada de información cuando se realiza un registro de desarrollador, con un dominio establecido para crear y alojar una aplicación web que use la información de la plataforma.

Los paquetes de datos vienen limitados a 30 entradas de datos y en el momento no se pueden hacer consultas históricas (las solicitudes de datos están restringidas al día en que se realizan). Las respuestas de datos vienen en formato no estructurado. JSON (JavaScript Object Notation) y contienen el texto, el nombre de usuario, número de seguidores, likes al comentario entre otras.

\subsection{Quandl}

Quandl es una fuente líder de datos financieros y económicos. A través de su API de consulta es bastante simple consultar series históricas de datos de las acciones de interés, usando curl en la línea de comandos o a través de un script en $\mathrm{R}$ o Python. A través de esta se obtienen los datos de las acciones de interés para este trabajo, y así generar la variación porcentual diaria del activo, objetivo final a predecir con este trabajo.

Al igual que la API de Stocktwits, las respuestas se obtienen en formato JSON, sin embargo esta permite también recibir un archivo en formato plano CSV o XML.

\section{Modelo de procesamiento de lenguaje natural}

El desarrollo de este trabajo se llevará a cabo siguiendo el enfoque empírico y estadístico del NLP. Como se mencionó anteriormente, este se cimenta en la conformación de un "corpus" o cuerpo de textos, sobre el cual se aplica una metodología o algoritmo que permita automatizar un análisis semántico de los textos, más específicamente relacionado con el sentimiento de los mismos. Para lograr esto, la máquina debe ser capaz de extraer la mayor cantidad de información de los textos ya previamente calificados para luego utilizar un modelo de Machine Learning diseñado específicamente para clasificar textos a los cuales no se les conoce su polaridad, ya sea positivos, neutros o negativos o en textos que expresan comentarios referentes a tendencias al alza o a la baja de un activo en el mercado de valores (semántica). A continuación se documenta paso a paso 
el procedimiento (resumido en la figura 12) llevado a cabo para generar los datos necesarios para el entrenamiento del modelo.

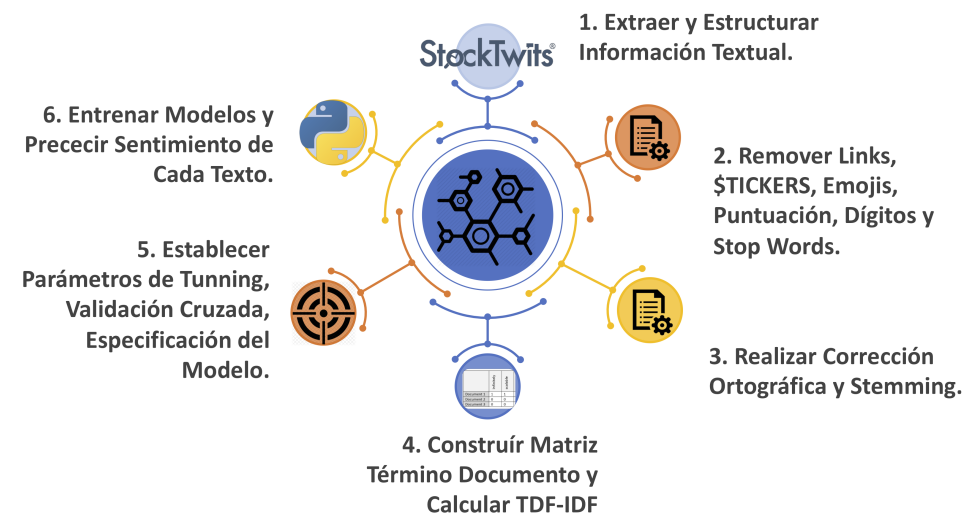

Figura 12: Diagrama de Modelo de Procesamiento de Lenguaje Natural Para Entrenamiento del Modelo, Elaboración Propia.

\subsection{Construcción del Corpus}

Dado que los datos no vienen estructurados o en una base de datos con variables definidas, es necesario procesarlos para crear una base de datos y de ella un cuerpo de textos con cada una de sus características (etiquetas de nivel de sentimiento). Para esta tarea existe un método llamado "Web Scrapping" mediante el cual el usuario, valiéndose de la estructura del código HTML o JSON genera una estructura unificada para los datos, sobre la cual se crea el corpus de textos para entrenar el modelo.

\subsection{Pre procesamiento del texto}

Cuando se trabaja con texto sin procesar, particularmente cuando se extrae de la web, no todo el texto es de utilidad o provee información importante que permita identificar su semántica. Elementos de un texto corto, comentario o tweet pueden contener enlaces, menciones, emojis, marcas de retweet, entre otras, que solo introducen ruido al modelo y hacen que sea menos efectivo. A parte de estos elementos, existen también algunos que siendo propios del lenguaje natural, generan ruido en el modelo. Este es el caso de las "Stop Words" y las "Function Words", las primeras son palabras que son usadas con demasiada frecuencia y las segundas son las que tienen como objetivo crear relaciones gramaticales y carecen de un significado intrínseco (cómo y, o, e, en español o or, and, yes, no en ingles). Dada la naturaleza de estas, con una alta probabilidad; estarán presentes en todos los textos por lo que no caracterizan el significado literal o carga de sentimiento de un texto.

Finalmente, en idiomas como el inglés y el español; se hace uso extensivo de signos de puntuación, variaciones entre mayúsculas y minúsculas, números y signos de exclamación para darle diferentes significados a un texto. Sin embargo, la máquina entiende cada conjunto de caracteres separado por un espacio o signo de puntuación como una palabra, aislando dichos signos como una "palabra" individual, que al estar presente en todos los textos no genera información relevante por lo que debe ser eliminada. Las mayúsculas y minúsculas deben ser tratadas también al pre-procesar el 
texto, para la máquina "Casa" es una palabra totalmente distinta a "casa", por lo que deben ser procesadas para permanecer todas en minúscula o mayúscula según decida el usuario.

\subsection{Corrección ortográfica y lematización}

A pesar de que los datos son obtenidos de una red social en la que los usuarios deben expresar de manera concreta, y correcta sus ideas acerca de un tema específico, se esperaría que los textos carezcan de errores ortográficos y de redacción. Sin embargo, son cosas que pueden suceder y el error humano solo genera ruido al momento de entrenar un modelo capaz de extraer las características de un texto, luego de el pre procesamiento es necesario aplicar algoritmos de corrección ortográfica y lematización, generalmente basados en distancias entre palabras. La corrección ortográfica consiste en reemplazar las palabras mal escritas por su contraparte correctamente redactada y la segunda tiene como objetivo traer las palabras a su raíz original, que en términos más técnicos es traer una palabra "flexionada" a su "lema" original (corrían y corriendo son palabras flexionadas del lema correr).

\subsection{Matriz Término Documento}

La matriz término documento es una forma de codificar o volver numéricos datos de texto. Se construye dejando las palabras presentes en todos los textos del corpus, previamente procesado; en las columnas, y la frecuencia con que estas aparecen en cada uno de los documentos en las filas. Esta se construye asumiendo que cada tweet es un documento individual, y se crea un set de características o variables. El orden o contexto de las palabras no es analizado, pero se trabaja bajo el supuesto de que documentos con cierta clasificación, contienen cierta variedad de palabras, que son entradas del modelo y deben ser identificadas por el modelo de machine learning.

\subsection{Frecuencia Término Documento y Frecuencia Inversa del Documento}

La Frecuencia Término Documento y Frecuencia Inversa del Documento (TDF-IDF por sus siglas en inglés) están basadas en la matriz término documento, y su función es generar más información de la que genera la frecuencia del término en cada documento. Ambas constituyen una medida que tiene en cuenta también cuantas veces aparece una palabra en todos los documentos y evita la aparición excesiva de ceros cuando se trata con textos cortos. La Frecuencia Término Documento es equivalente a la ecuación (70):

$$
T D F(t, d)=\frac{f_{t, d}}{\sum_{i=1}^{D} f_{t, i}}
$$

En la cual el numerador representa el número de veces que aparece el término $t$ en el documento $d$ y el denominador es la suma de las frecuencias del término $t$ a través de los $i=1, \ldots, D$ documentos. Y la Frecuencia Inversa del Documento equivale a la ecuación (71):

$$
I D F(t, D)=\log \frac{N}{|\{d \in D: t \in d\}|}
$$

Donde el numerador es el número de documentos del corpus y el denominador es el número de documentos del corpus en los que aparece el término $t$. Finalmente la medida TDF-IDF se compone de la multiplicación entre el $T D F(t, d)$ y el $I D F(d, D)$. 


\section{Metodología de predicción}

Uno de los objetivos de este trabajo es proponer una nueva metodología de aprovechamiento de los datos de la red social para lograr la predicción de la dirección de variación del precio de apertura y de cierre del activo. (Coyne et al, 2017) en su artículo prueba diferentes metodologías y ventanas de tiempo para lograr las predicciones comparándolas según su precisión promedio. Para este trabajo se propone una metodología que busca integrar la idea del "Smart User" y aprovechar la información del sentimiento del mercado y la ponderación que le dan los usuarios a los comentarios según su sentimiento (proporción de likes por tipo de tweet).

Ahora bien, el aprovechamiento de la información extraída de la red social StockTwits se realiza mediante dos procesos paralelos (resumidos en la figura ??) que luego se juntan para generar la información que permite el entrenamiento del modelo y la predicción.

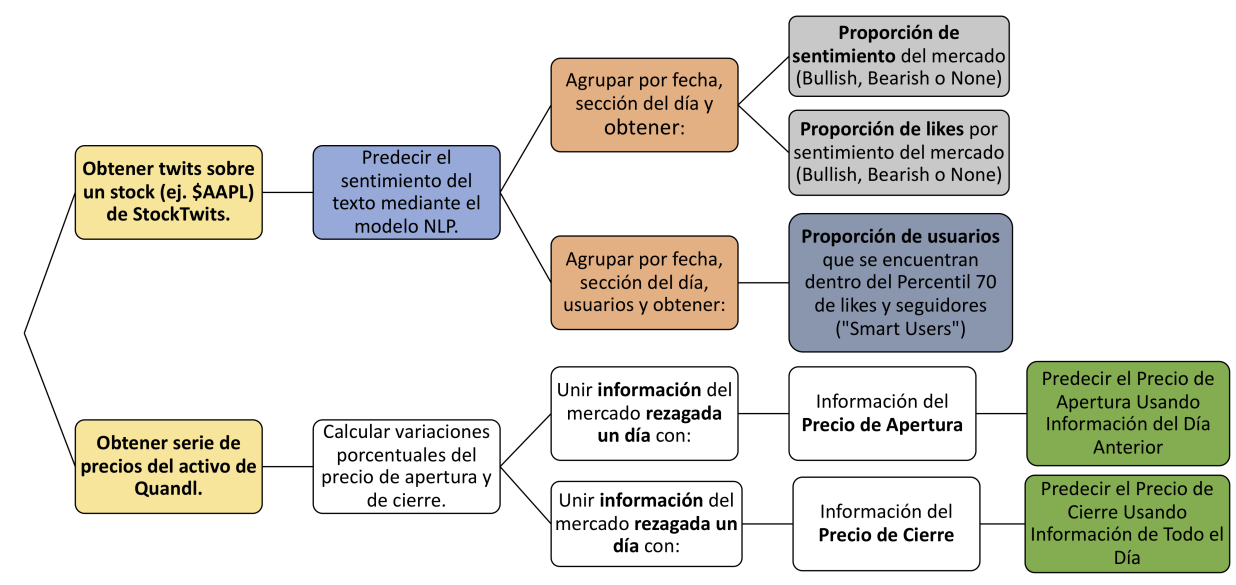

Figura 13: Diagrama de Metodología de Predicción del la Dirección de Variación del Precio, Elaboración Propia.

El primero se logra aplicando el modelo de procesamiento del lenguaje natural (previamente entrenado con un conjunto de textos calificados) para obtener el sentimiento de los textos en el conjunto de datos. Hay que resaltar, que se eliminan los textos que no expresan sentimiento alguno, es decir la categoría None, dado que no aportan información relevante para el modelo de NLP y existe un alto porcentaje de ellos en los datos extraídos. Una vez calificados los textos, se obtiene el sentimiento general del mercado; agrupando los datos por fecha y obteniendo la proporción de textos que hablan de tendencias alcistas ("bullish") y bajistas ("bearish"). Se obtiene también el nivel de aceptación que le dan los usuarios a lo que expresa el mercado en general, es decir, se agrupa la información por días y se calcula la proporción de likes que reciben los comentarios según la tendencia que expresan.

Como último paso del primer proceso, se busca cuantificar la relevancia que le están dando los usuarios destacados al debate sobre la acción. Para medir dicha relevancia se agrupa la información por días y por usuarios, para identificar cuantos seguidores tienen los usuarios en ese momento del tiempo y cuantos "likes" han recibido sus comentarios ese día en particular. Los usuarios que se encuentren arriba del percentil 70 del número de likes y del percentil 70 de número de seguidores, son marcados ese día como "Smart Users". De la marca de Usuario Relevante; se obtiene el porcentaje de usuarios "Smart" que se encuentran activos hablando sobre esa acción en ese 
momento del tiempo como variable de cuantificación de la relevancia que se le está dando al debate sobre el activo en ese momento del tiempo.

El otro proceso tiene que ver con la obtención de los datos del precio de la acción y su consolidación para crear una base de datos apropiada para el entrenamiento del modelo. Por este lado de la metodología, se inicia realizando la descarga de los precios de apertura y cierre del activo mediante la plataforma Quandl, obteniendo la variación porcentual de los mismos de un día a otro y marcando la variación como "Up" si la variación porcentual es negativa o "Down" si es negativa.

Luego, con la dirección de variación tanto de los precios de apertura como de cierre se une la información de tal manera que los datos agrupados obtenidos de ayer permitan predecir la dirección de variación del precio de apertura del día de mañana y, que los datos obtenidos de la red social el día de hoy sean insumo para la predicción de la dirección de variación del precio de cierre del mismo día.

\section{Resultados y discusión}

En este apartado se muestran los rendimientos obtenidos par cada uno de los modelos, para la automatización del etiquetado del sentimiento general de los textos (bullish o bearish) y la predicción de la dirección de variación del precio ("Up" o "Down"). Los modelos serán comparados de la siguiente manera:

- Para el Etiquetado Automático de Sentimiento, se compararán las precisiones promedio; realizando validación cruzada de particiones aleatorias (incluyendo una semilla para replicabilidad) o "K-Fold Cross Validation"de 5 folds con los datos de entrenamiento, iterando sobre un intervalo $(0,1)$ de parámetros de penalización L2 para la red neuronal y sobre la profundidad máxima de los árboles para los predictores Random Forest y XGBoost. Una vez identificado el parámetro que maximiza la precisión promedio se calculará la curva ROC y su respectiva área bajo la curva ( $A U C$ por sus siglas en inglés), y la curva de *Precisión versus Recall*, con los datos de test.

- Para la Predicción de la Dirección de la Variación del Precio, se compararán las precisiones promedio; realizando validación cruzada, de particiones aleatorias (incluyendo una semilla para replicabilidad) o " $K$-Fold Cross Validation"de 5 folds con los datos de entrenamiento. Al igual que en el modelo de NLP se iterará sobre un conjunto de parámetros de penalización L2 para la red neuronal y sobre la profundidad máxima de los árboles para los demás algoritmos. Una vez encontrado el parámetro que maximiza la precisión promedio se calculará la curva ROC y la curva de *Precisión versus Recall*, sobre los datos de validación junto con su respectiva área bajo la curva o AUC como criterio adicional de decisión.

Además, todos los modelos y validaciones cruzadas fueron realizadas con una semilla para la generación de pesos iniciales y particiones de test y validación, garantizando así su completa replicabilidad sobre los datos obtenidos.

\subsection{Etiquetado Automático del sentimiento de los Textos}

Como se mencionó en la introducción a este apartado, se mostrara a detalle la configuración de los modelos que generaron mayores rendimientos y además una serie de gráficos de resultados de validación de los predictores propuestos, seleccionados para automatizar el etiquetado del sentimiento (Random Forest, XGboost y Redes Neuronales). Los datos corresponden a 144.809 textos cortos extraídos de la plataforma StockTwits entre el 11 de octubre y el 11 de noviembre de 2018, de los cuales se usaron 39.813 (eliminando 104.996 de la categoría "None"). Para el entrenamiento del modelo se usa una muestra aleatoria simple estratificada según la cantidad de 
textos por tipo de sentimiento del $60 \%$ del total de datos extraídos. Al construir la matriz término documento, se conservan las palabras con una frecuencia mínima de 29 por lo que se tienen 479 variables o palabras.

\subsubsection{Random Forest}

El predictor Random Forest para el modelo NLP fue configurado con 100 árboles de clasificación, usando el criterio de entropía para la división de los nodos y una profundidad máxima (numero máximo de variables a usar en el crecimiento del árbol) de 479, identificada como la que maximiza la precisión promedio del modelo en la validación cruzada, en el panel izquierdo de la figura 14 se observa como la precisión aumenta a medida que la profundidad lo hace y llega a un valor máximo de 0.754637 cuando la profundidad es mayor o igual a 98 .
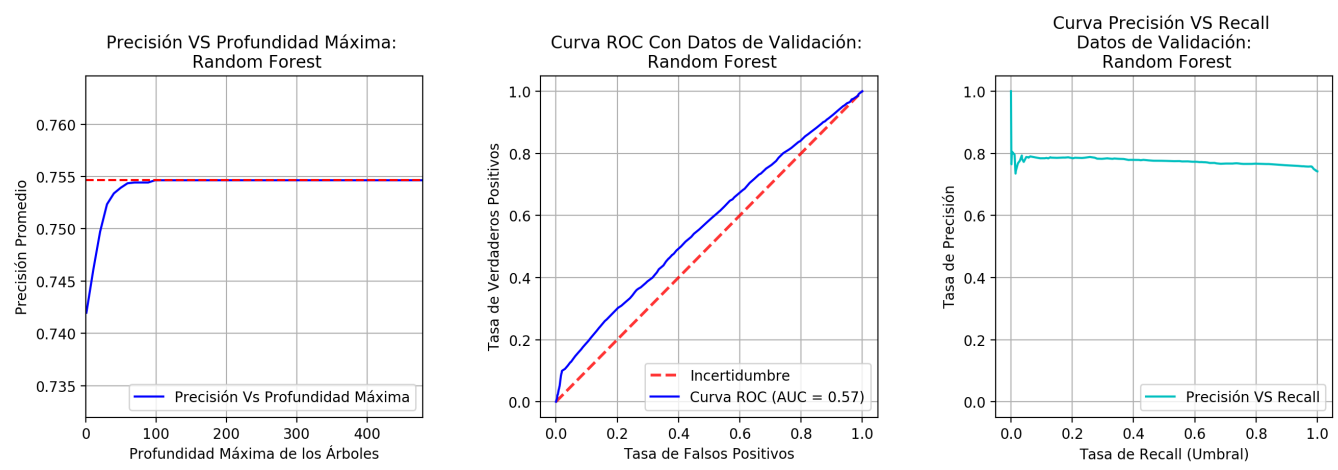

Figura 14: Validación predictor Random Forest para NLP, Elaboración Propia.

En los paneles medio y derecho de la figura 14 se evidencia que, a pesar de que la curva ROC a penas logra separarse de la línea de incertidumbre, mostrando que el modelo no ofrece una ganancia substancial de verdaderos positivos a un costo de verdaderos negativos (consecuencia de la inestabilidad que caracteriza a los predictores basados en árboles o redes neuronales); el predictor se muestra estable ante variaciones en el Recall o umbral de decisión del clasificador, evidenciando la capacidad del ensamblaje de árboles para aprovechar la inestabilidad de un predictor y convertirla en mayor precisión.

Cuadro 3: Matriz de Confusión, Modelo NLP usando Random Forest

\begin{tabular}{|l|l|l|l|}
\cline { 3 - 4 } \multicolumn{2}{c|}{} & \multicolumn{2}{l|}{ Clase Real } \\
\cline { 3 - 4 } \multicolumn{2}{c|}{} & Bearish & Bullish \\
\hline \multirow{2}{*}{ Predicción } & Bearish & 307 & 2775 \\
\cline { 2 - 4 } & Bullish & 175 & 8687 \\
\hline
\end{tabular}

Elaboración propia.

La tabla de confusión (3), del modelo Random Forest; muestra como correctamente logra identificar 307 textos que hablan de tendencias bajistas y 8687 textos que muestran tendencia al alza usando de los datos de validación; lo que representa una precisión del $75.302 \%$. 


\subsubsection{XGBoost}

En el predictor de Xtreme Gradient Boosting, el panel izquierdo de la figura 15 muestra como el parámetro de profundidad de los árboles logra maximizar la precisión promedio de la validación desde las 14 variables y desde allí se mantiene constante. Al igual que el predictor basado en el algoritmo Random Forest el modelo parece no ser estable y su curva ROC a penas se despega de la línea de incertidumbre, pero logra mantener la precisiones muy cercanas al $80 \%$ a pesar de variaciones den el umbral de decisión del modelo, consecuencia del "Boosting" de gradiente.
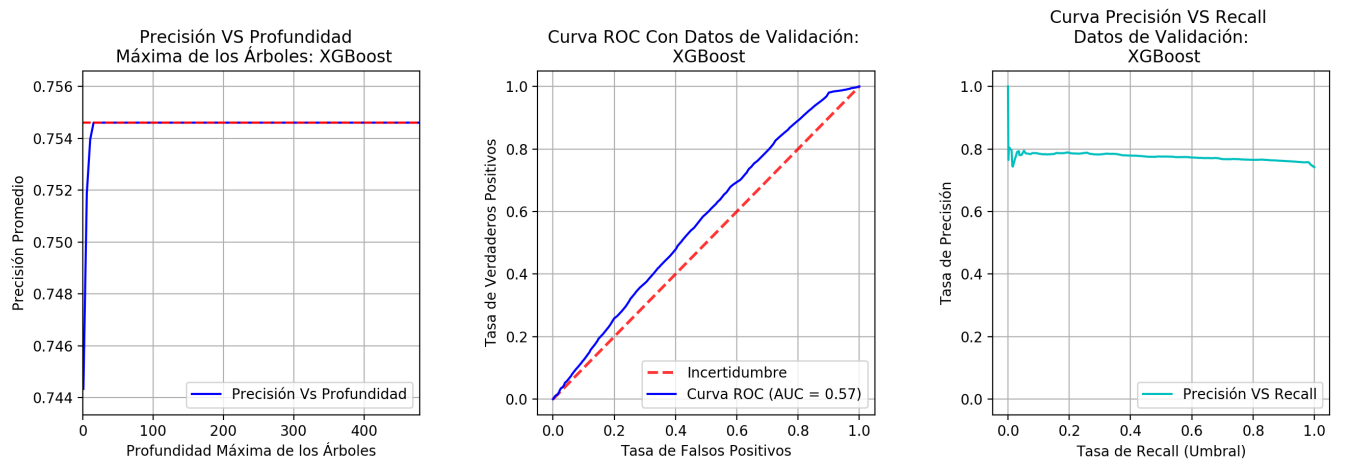

Figura 15: Validación predictor XGBoost para NLP, Elaboración Propia.

Para este caso, el predictor XGBoost fue configurado con una profundidad máxima de 479 variables para la construcción de los árboles de clasificación mediante el algoritmo "Greedy", usando como métrica de evaluación de rendimiento el área bajo la curva de la curva ROC.

Cuadro 4: Matriz de Confusión, Modelo NLP usando XGBoost

\begin{tabular}{|l|l|l|l|}
\cline { 3 - 4 } \multicolumn{2}{c|}{} & \multicolumn{2}{|l|}{ Clase Real } \\
\cline { 3 - 4 } \multicolumn{2}{c|}{} & Bearish & Bullish \\
\hline \multirow{2}{*}{ Predicción } & Bearish & 307 & 2775 \\
\cline { 2 - 4 } & Bullish & 175 & 8687 \\
\hline
\end{tabular}

Elaboración propia.

La tabla de confusión (4), del modelo XGBoost; muestra como correctamente logra identificar 307 textos que hablan de tendencias bajistas y 8687 textos que muestran tendencia al alza usando de los datos de validación; lo que representa una precisión del $75.302 \%$.

\subsubsection{Redes Neuronales}

El predictor de redes neuronales artificiales fue configurado con 7 capas ocultas en forma de mariposa con $60,30,10,2,10,30$ y 60 unidades de procesamiento cada una. La función de activación es la sigmoide de tangente hiperbólica, un máximo de iteraciones de 300 y una tolerancia de 0.00001 como criterio de parada de las iteraciones ante ningún cambio en la pérdida mínima asociada a la estimación de los pesos. La influencia de la penalización L2 durante la validación cruzada tiene influencia sobre la precisión promedio, logrando una precisión máxima de 0.7549 (panel izquierdo de la figura 16). 

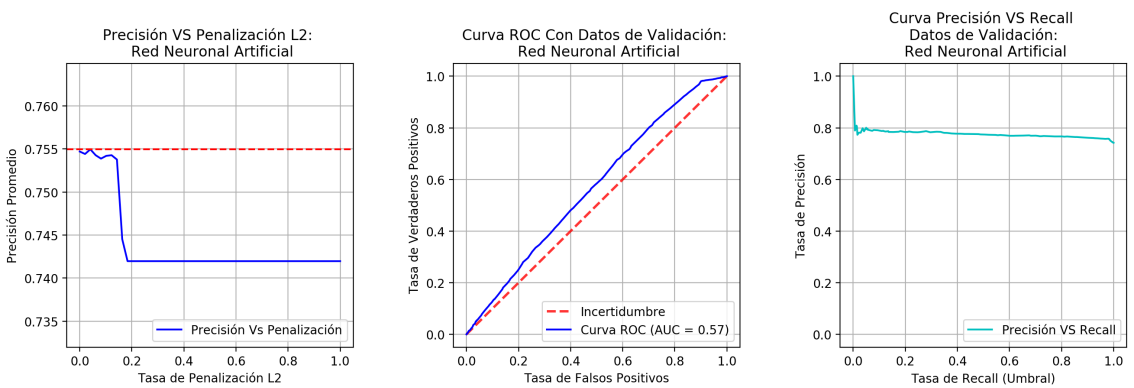

Figura 16: Validación predictor de Redes Neuronales Artificiales para NLP, Elaboración Propia.

El comportamiento de la curva ROC es similar al de los anteriores predictores debido a la inestabilidad, sin embargo, la red neuronal logra las tasas más altas de precisión ante cambios en el umbral de decisión lo que permite lograr buenas predicciones a pesar de la alta varianza, tal y como se evidencia en los paneles central y derecho de la figura 16.

Cuadro 5: Matriz de Confusión, Modelo NLP usando red neuronal

\begin{tabular}{|l|l|l|l|}
\cline { 3 - 4 } \multicolumn{2}{c|}{} & \multicolumn{2}{|l|}{ Clase Real } \\
\cline { 3 - 4 } \multicolumn{2}{c|}{} & Bearish & Bullish \\
\hline \multirow{2}{*}{ Predicción } & Bearish & 299 & 2783 \\
\cline { 2 - 4 } & Bullish & 168 & 8694 \\
\hline
\end{tabular}

Elaboración propia.

La tabla de confusión (5), del modelo de redes neuronales; muestra como correctamente logra identificar 299 textos que hablan de tendencias bajistas y 8694 textos que muestran tendencia al alza usando de los datos de validación lo que representa una precisión del $75.293 \%$.

\subsection{Predicción de la dirección de variación del precio}

Para la predicción de la dirección de la variación del precio de las acciones, se tienen datos descargados desde el 11 de octubre al 4 de diciembre de 2018. La información del sentimiento del mercado es resumida tal y como se muestra en la metodología de aprovechamiento de la información propuesta en esta investigación, calculando el sentimiento general del mercado (proporción diaria de textos alcistas y bajistas), el porcentaje de aprobación que se le da a este (porcentaje de likes que recibe cada tipo de sentimiento), y la relevancia de la discusión (porcentaje de usuarios "smart").

Para el entrenamiento de los modelos de cada acción se toma la información descargada desde el 11 de octubre hasta el 18 de noviembre de 2018, dejando dos semanas y cuatro días para la validación de los modelos. Dado que la información debe ser resumida a un indicador diario, y la plataforma para desarrolladores de StockTwits no permite hacer descargas históricas; la información de entrenamiento es ampliada mediante simulaciones, realizando muestreo aleatorio simple expansivo, con reemplazamiento, para obtener un set de datos de entrenamiento de $10 \mathrm{mil}$ observaciones. Los resultados de cada modelo son mostrados a continuación. 


\subsubsection{Acciones de Apple Inc.(AAPL)}

\subsubsection{Random Forest}

Para la acción de AAPL se configuraron los árboles con una profundidad máxima de 6 variables (profundidad que logra la precisión promedio máxima según el panel izquierdo de la figura 17), 200 árboles de clasificación y función de entropía para la división de los nodos.
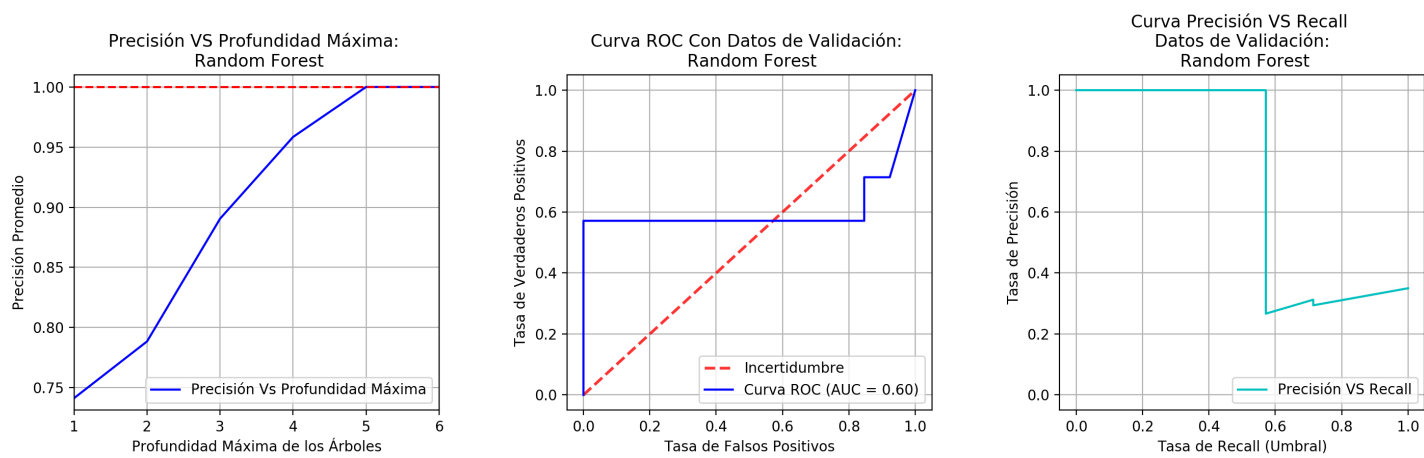

Figura 17: Validación predictor Random Forest para la dirección de variación del precio de la acción de Apple Inc., Elaboración Propia.

La curva ROC se mantiene correcta para tasas de verdaderos positivos hasta del $60 \%$ sin representar un costo de una más alta tasa de falsos positivos, pero su costo se incrementa cuando genera tasas de verdaderos positivos por encima del $60 \%$, siendo este mayor en todos los puntos (panel central de la figura 17). El comportamiento de la curva ROC se ve reflejado también el la curva de precisión versus recall (panel derecho de la figura 17) que muestra como el predictor se mantiene estable cuando el umbral de decisión es cercano a 0.6, estabilidad que se pierde cuando el umbral supera este valor.

Cuadro 6: Matriz de Confusión, modelo para APPL usando Random Forest

\begin{tabular}{|l|l|l|l|}
\cline { 3 - 4 } \multicolumn{2}{c|}{} & \multicolumn{2}{|c|}{ Clase Real } \\
\cline { 3 - 4 } \multicolumn{2}{c|}{ Predicción } & Down & Up \\
\cline { 2 - 4 } & Up & 3 & 2 \\
\hline
\end{tabular}

Elaboración propia.

En la matriz de confusión sobre los datos de validación se observa como el predictor Random forest predice correctamente 13 variaciones porcentuales negativas del precio y 4 positivas; logrando una precisión de $77.27 \%$.

\subsubsection{XGBoost}

El modelo XGBoost para este activo, quedó configurado con una profundidad máxima de 6 variables para la construcción de los árboles, dado que esta maximiza la precisión máxima promedio durante la validación cruzada (panel izquierdo de la figura 18). 

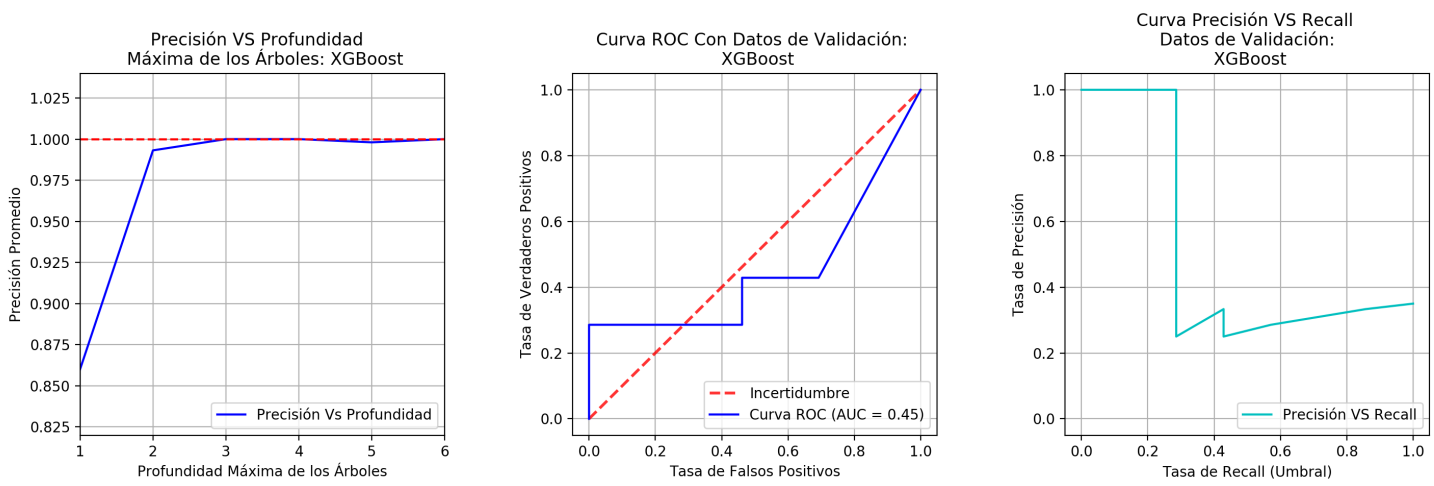

Figura 18: Validación predictor XGBoost para la dirección de variación del precio de la acción de Apple Inc., Elaboración Propia.

A pesar de esta configuración, en el panel central y derecho de la figura 18 se evidencia como modelo no se comporta correctamente sobre los datos de validación; la mayoría de los puntos de la curva ROC se posicionan debajo de la línea de incertidumbre y la precisión se ve altamente afectada ante una variación en el umbral de decisión del predictor, indicando que la precisión se maximiza en la muestra de validación únicamente con umbrales cercanos a cero.

Cuadro 7: Matriz de Confusión, modelo para APPL usando XGBoost

\begin{tabular}{|c|c|c|c|}
\hline & \multicolumn{2}{|c|}{ Clase Real } \\
\hline & & Down & Up \\
\hline \multirow{2}{*}{ Predicción } & Down & 14 & 1 \\
\hline & Up & 5 & 2 \\
\hline
\end{tabular}

Elaboración propia.

La inestabilidad del modelo es consecuencia de su inestabilidad, sin embargo, el ensamblaje y "boosting" permiten que logre una precisión de $72.72 \%$ sobre los datos de validación (dada la matriz de confusión de la tabla 7)

\subsubsection{Redes Neuronales}

El modelo de redes neuronales para esta acción se configura con un parámetro de penalización L2 de 0.95918367, el cual maximiza la precisión promedio durante la validación (panel izquierdo figura 19). La red tiene dos capas ocultas de 7 y 2 unidades de procesamiento respectivamente, cada una con función logística de activación, el número máximo de iteraciones sobre los datos es de 300 con una tolerancia ante la convergencia de la pérdida mínima de 0.0001 . 

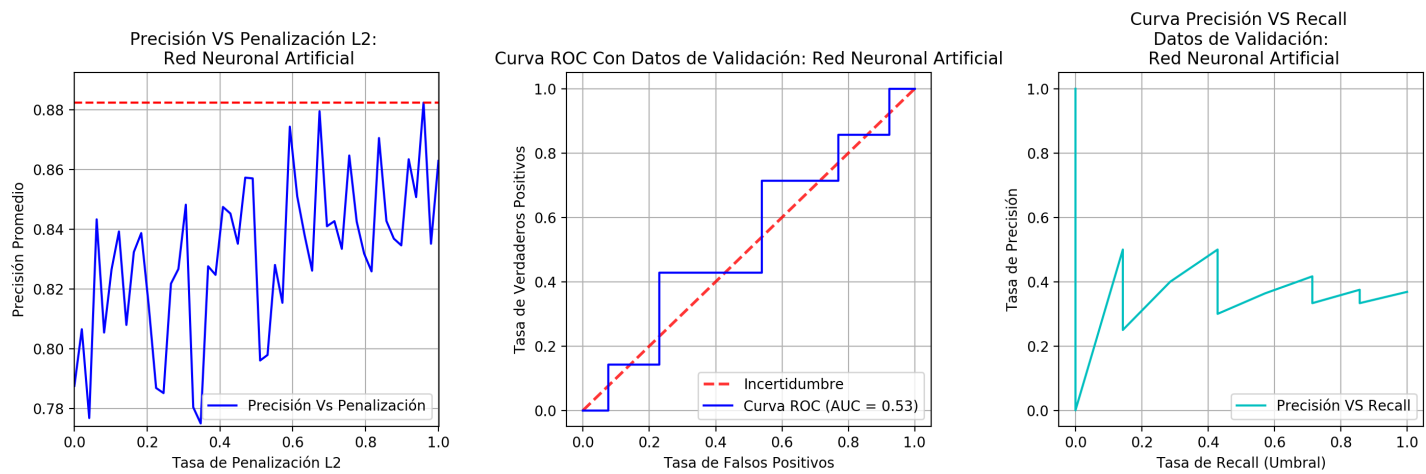

Figura 19: Validación predictor de Redes Neuronales Artificiales para la dirección de variación del precio de la acción de Apple Inc., Elaboración Propia.

La curva ROC, y la curva de precisión versus recall no tienen buen comportamiento al ser calculadas sobre los datos de test, a pesar de la penalización. Con nuevos datos este modelo no logra tasas de verdaderos positivos y falsos negativos lejanas de la línea de incertidumbre y la tasa de precisión decae rápidamente ante variaciones simples en el umbral de decisión.

Cuadro 8: Matriz de Confusión, modelo para APPL usando red neuronal

\begin{tabular}{|l|l|l|l|}
\cline { 3 - 4 } \multicolumn{2}{c|}{} & \multicolumn{2}{|c|}{ Clase Real } \\
\cline { 3 - 4 } \multicolumn{2}{c|}{ Down } & Up \\
\hline \multirow{2}{*}{ Predicción } & Down & 11 & 4 \\
\cline { 2 - 4 } & Up & 5 & 2 \\
\hline
\end{tabular}

Elaboración propia.

La matriz de confusión muestra como el modelo logra predecir correctamente 11 caídas del precio de la acción de AAPL y dos subidas; logrando una precisión de $59.1 \%$ sobre los datos de validación.

\subsubsection{Acciones de International Business Machines Corporation (IBM)}

\subsubsection{Random Forest}

El predictor Random Forest para la acción de IBM se configuró con una profundidad máxima de 5 variables, dado que esta maximiza la precisión promedio durante la validación (panel izquierdo figura 20), usando 200 árboles y función de entropía para la partición de los nodos. 

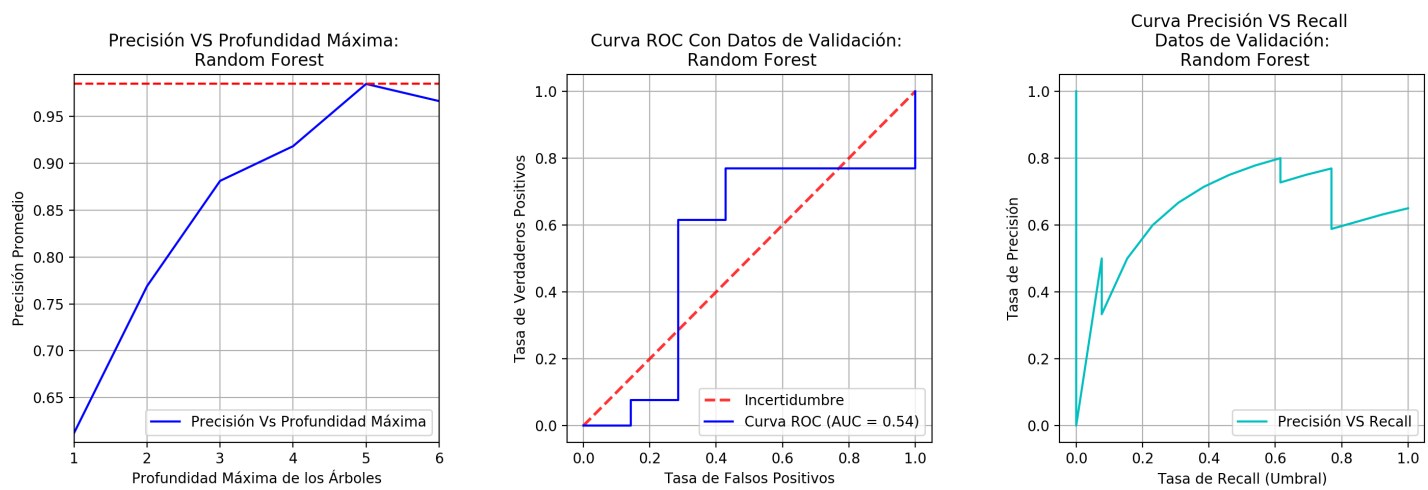

Figura 20: Validación predictor Random Forest para la dirección de variación del precio de la acción de IBM, Elaboración Propia.

La curva ROC se mantiene arriba de la línea de incertidumbre para tasas de verdaderos positivos y falsos negativos entre el $40 \%$ y $80 \%$, valor en el que logra mejor rendimiento sin generar estabilidad. A partir de tasas de verdaderos positivos superiores al $80 \%$ los falsos positivos aumentan a $100 \%$. Este comportamiento de la curva ROC se ve reflejado en el crecimiento de la precisión ante variaciones en el umbral de decisión de 0.6, un umbral superior implica una caída de la precisión.

Cuadro 9: Matriz de Confusión, modelo para IBM usando Random Forest

\begin{tabular}{|l|l|l|l|}
\cline { 3 - 4 } \multicolumn{2}{c|}{} & \multicolumn{2}{|c|}{ Clase Real } \\
\cline { 3 - 4 } \multicolumn{2}{c|}{} & Down & Up \\
\hline \multirow{2}{*}{ Predicción } & Down & 5 & 4 \\
\cline { 2 - 4 } & Up & 10 & 3 \\
\hline
\end{tabular}

Elaboración propia.

La matriz de confusión muestra como el modelo logra predecir correctamente 3 variaciones porcentuales positivas de la acción y 5 negativas, logrando a penas una precisión del $36.36 \%$ sobre los datos de validación.

\subsubsection{XGBoost}

El predictor XGBoost para este activo se entrena con una profundidad máxima de 6 variables para la construcción de los árboles, dado que estas maximizan la precisión promedio de la validación cruzada (panel izquierdo de la figura 21). 

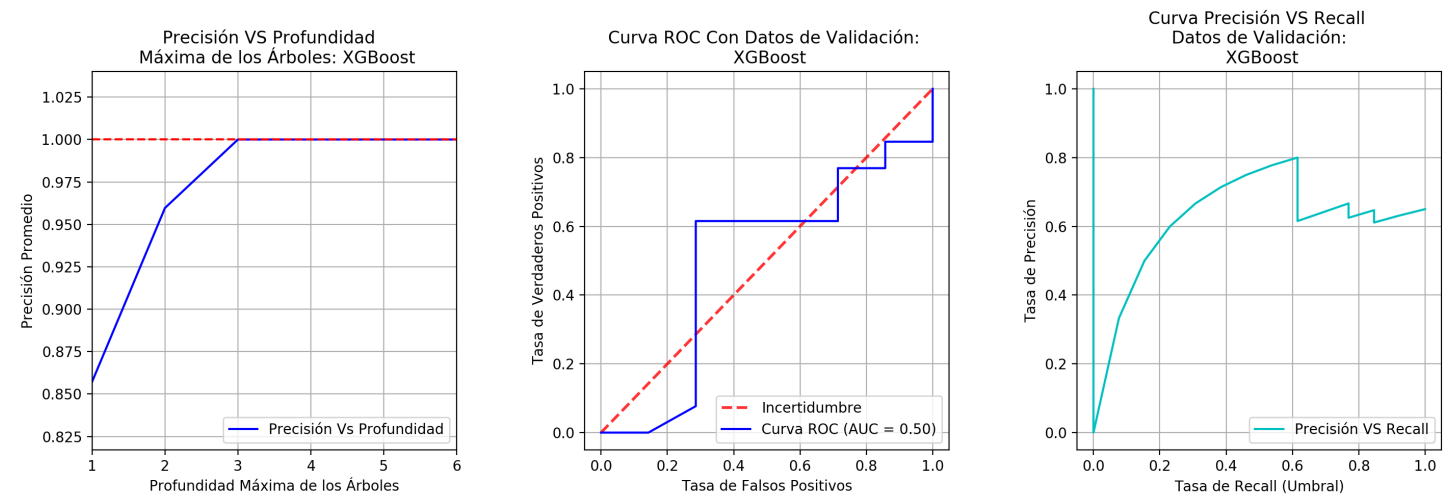

Figura 21: Validación predictor XGBoost para la dirección de variación del precio de la acción de IBM, Elaboración Propia.

La curva ROC y la precisión versus recall indican una alta inestabilidad del modelo frente a su evaluación sobre los datos de validación (panel central y derecho de la figura 21). Según la primera curva, el modelo solo logra superar la línea de incertidumbre hasta tasas de verdaderos positivos del $60 \%$, para valores superiores los falsos positivos superan a los verdaderos, comportamiento que es evidente en la tercera curva, que muestra estabilidad únicamente en un umbral de decisión de 0.6 .

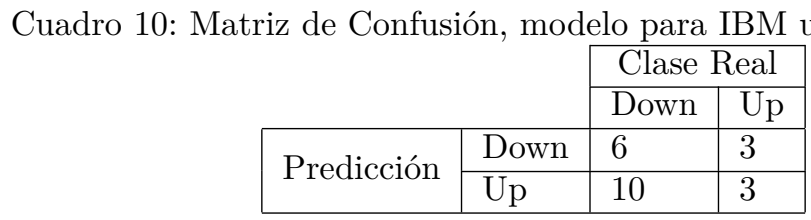

Elaboración propia.

La matriz de confusión muestra como el modelo logra predecir correctamente 3 variaciones porcentuales positivas de la acción y 6 negativas, logrando a penas una precisión del 40.9\% sobre los datos de validación.

\subsubsection{Redes Neuronales}

El predictor de redes neuronales para esta acción tiene dos capas ocultas de 6 y 2 unidades de procesamiento con función de activación logística en cada una, el número máximo de iteraciones es de 300 con una tolerancia de no disminución de la pérdida de 0.0001. Los pesos de conexión tienen un parámetro de penalización L2 de 0.91836735, pues según el panel izquierdo de la figura 22; logran la precisión promedio máxima durante la validación. 

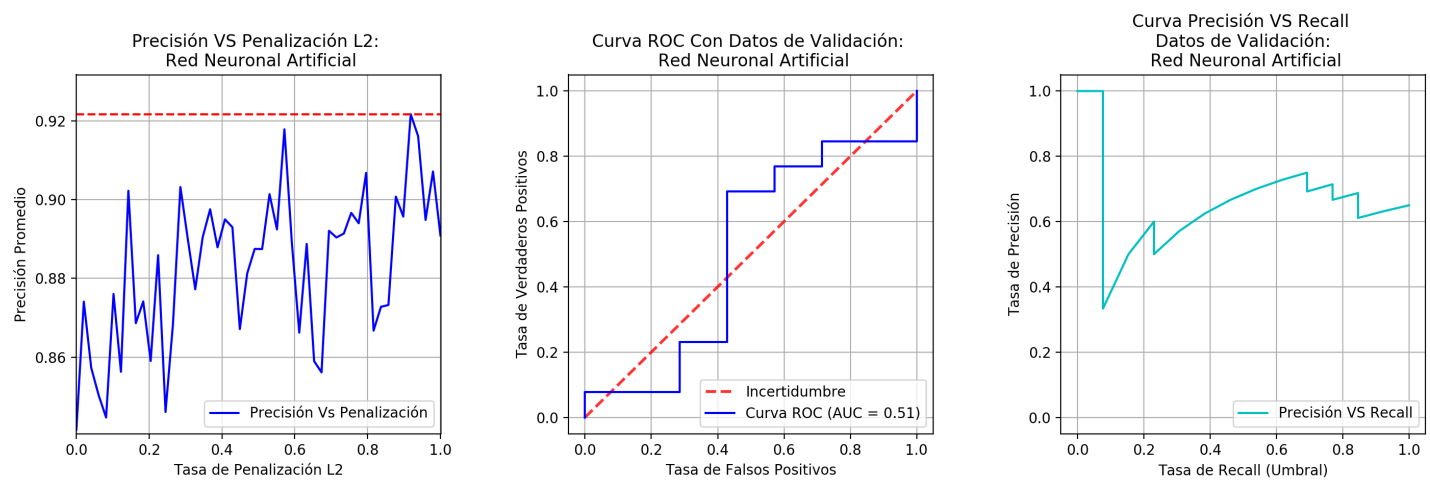

Figura 22: Validación predictor de Redes Neuronales Artificiales para la dirección de variación del precio de la acción de IBM, Elaboración Propia.

La curva ROC sobre los datos de validación, muestra un rendimiento pobre del modelo con los datos de validación, pues esta supera en un pequeño tramo a la curva de incertidumbre (panel central de la figura 22). A pesar de esto, el predictor logra cierta estabilidad ante cambios en el umbral de decisión, manteniendo tasas de precisión entre el $60 \%$ y $80 \%$ ciando el umbral es superior a 0.4 (panel central de la figura 22).

Cuadro 11: Matriz de Confusión, modelo para IBM usando redes neuronales

\begin{tabular}{|l|l|l|l|}
\cline { 3 - 4 } \multicolumn{2}{c|}{} & \multicolumn{2}{|c|}{ Clase Real } \\
\cline { 3 - 4 } \multicolumn{2}{c|}{ Down } & Up \\
\hline \multirow{2}{*}{ Predicción } & Down & 4 & 5 \\
\cline { 2 - 4 } & Up & 8 & 5 \\
\hline
\end{tabular}

Elaboración propia.

La matriz de confusión muestra como el modelo logra predecir correctamente 5 variaciones porcentuales positivas de la acción y 4 negativas, logrando a penas una precisión del $40.91 \%$ sobre los datos de validación.

\subsubsection{Acciones de Microsoft Corporation (MSFT)}

\subsubsection{Random Forest}

El predictor Random Forest para la acción de MSFT se configuró con una profundidad máxima de 6 variables, dado que esta maximiza la precisión promedio durante la validación (panel izquierdo figura 20), usando 200 árboles y función de entropía para la partición de los nodos. 

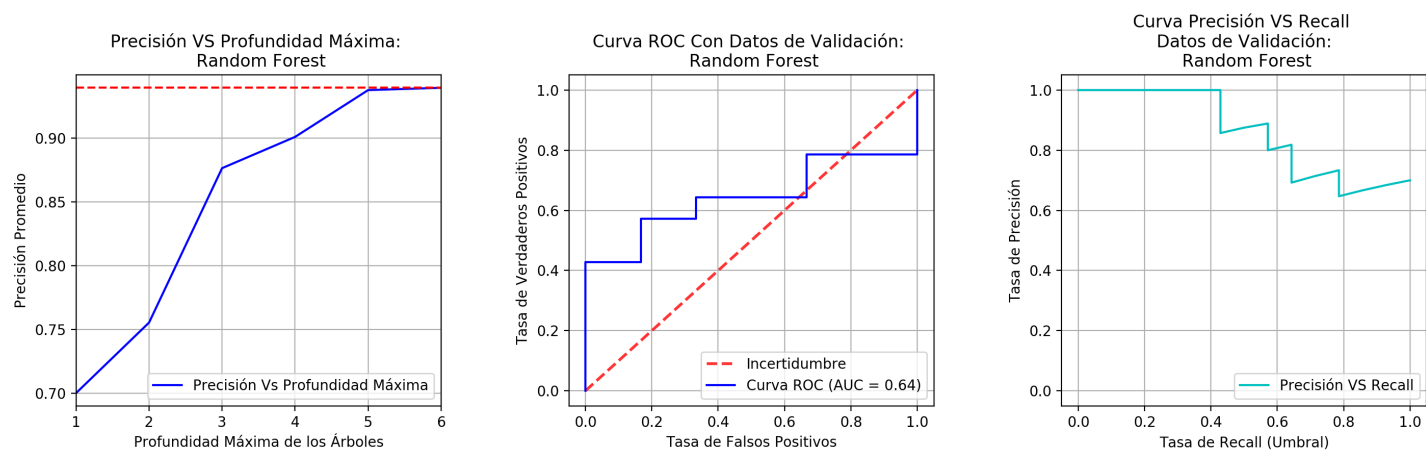

Figura 23: Validación predictor Random Forest para la dirección de variación del precio de la acción de Microsoft, Elaboración Propia.

La curva ROC se mantiene correcta para tasas de verdaderos positivos hasta del $80 \%$ sin representar un costo de una más alta tasa de falsos positivos, a partir de este valor el costo de falsos positivos es mayor tal y como lo muestra el panel central de la figura 17. A pesar de esto, la curva se comienza a acercar rápidamente a la línea de incertidumbre luego de una tasa de verdaderos positivos del $40 \%$, lo que explica el comportamiento de la curva del panel derecho de la figura 17; en la que se observa como la precisión cae de $100 \%$ a $60 \%$ cuando el umbral de decisión es superior a 0.4.

Cuadro 12: Matriz de Confusión, modelo para MSFT usando Random Forest

\begin{tabular}{|c|c|c|c|}
\hline & \multicolumn{2}{|c|}{ Clase Real } \\
\hline & & Down & $\mathrm{Up}$ \\
\hline \multirow{2}{*}{ Predicción } & Down & 7 & 1 \\
\hline & $\mathrm{Up}$ & 8 & 6 \\
\hline
\end{tabular}

Elaboración propia.

La matriz de confusión muestra como el modelo logra predecir correctamente 6 variaciones porcentuales positivas de la acción y 7 negativas, logrando a penas una precisión del $59.09 \%$ sobre los datos de validación.

\subsubsection{XGBoost}

El predictor de XGBoost para esta acción tiene 6 variables como profundidad máxima, dado que esta maximiza la precisión promedio durante la validación (panel izquierdo figura 24).
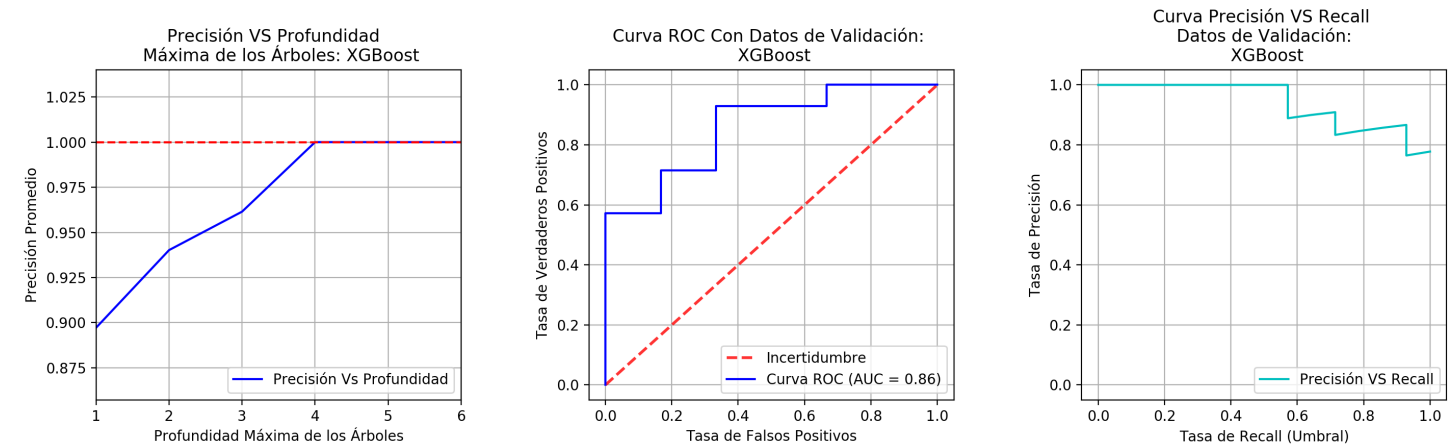

Figura 24: Validación predictor XGBoost para la dirección de variación del precio de la acción de Microsoft, Elaboración Propia. 
La curva ROC (panel central figura 24) tiene un comportamiento muy bueno al evaluarla sobre los datos de validación, se mantiene en su totalidad sobre la línea de incertidumbre, lo que significa generación de verdaderos positivos a costo de tasas más bajas de falsos positivos. La curva del panel derecho de la figura 24 muestra la estabilidad del predictor ante cambios en el umbral de decisión, un umbral superior a 0.5 implica una disminución de la precisión, sin embargo, esta reducción no implica precisiones inferiores al $75 \%$

Cuadro 13: Matriz de Confusión, modelo para MSFT usando XGBoost

\begin{tabular}{|c|l|l|l|}
\cline { 3 - 4 } \multicolumn{2}{c|}{} & \multicolumn{2}{c|}{ Clase Real } \\
\cline { 3 - 4 } \multicolumn{2}{c|}{ Predicción } & Down & Up \\
\cline { 2 - 4 } & Up & 6 & 2 \\
\cline { 2 - 4 }
\end{tabular}

Elaboración propia.

La matriz de confusión muestra como el modelo logra predecir correctamente 8 variaciones porcentuales positivas de la acción y 6 negativas, logrando a penas una precisión del $63.63 \%$ sobre los datos de validación.

\subsubsection{Redes Neuronales}

El modelo de redes neuronales para esta acción se compone de 2 capas ocultas cada una con 6 y 2 unidades de procesamiento con la función logística de activación. El número máximo de iteraciones al set de entrenamiento es de 300 con una tolerancia de parada ante mínimo cambio en la pérdida de 0.0001. Se incluye además un parámetro de penalización L2 de 0.53061224, debido a que maximiza la precisión promedio durante la validación (panel izquierdo de la figura 25).
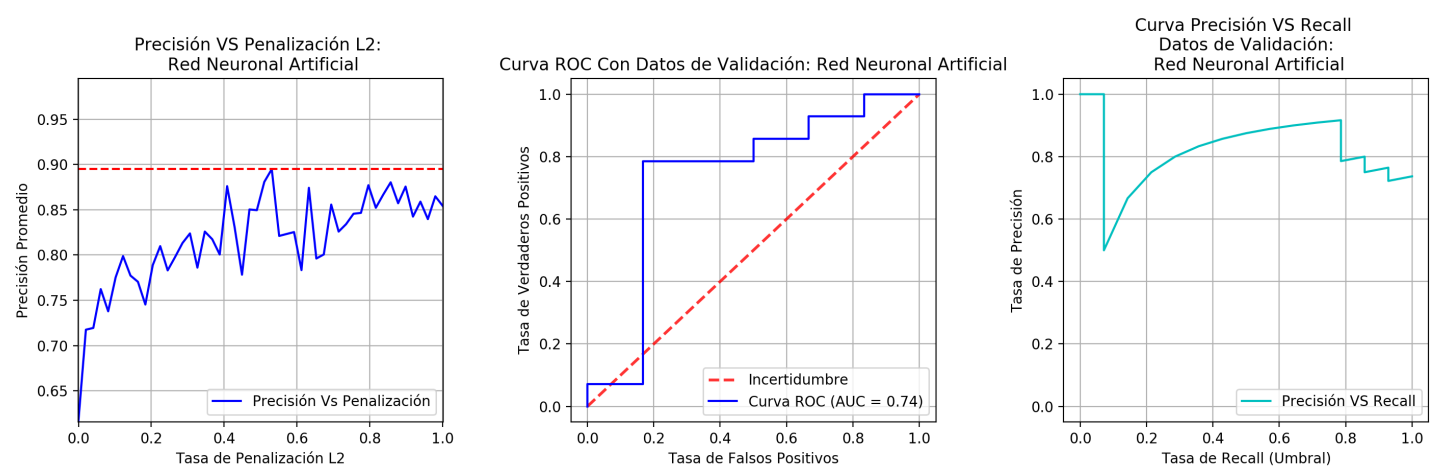

Figura 25: Validación predictor de Redes Neuronales Artificiales para la dirección de variación del precio de la acción de Microsoft, Elaboración Propia.

La curva ROC con los datos de validación muestra un comportamiento aceptable, dado que el modelo es capaz de lograr tasas de verdaderos positivos hasta del $80 \%$ a un costo de $20 \%$ de falsos positivos (panel central de la figura 25), sobre este rango se genera inestabilidad y el costo de generar verdaderos positivos casi iguala el de falsos positivos en la parte superior de la curva. La inestabilidad de la curva ROC se ve reflejada en el panel derecho de la figura \{img:msft_redneuronal\}, en la cual la precisión promedio crece hasta que el límite de decisión del modelo llega a 0.8 donde decae. 
Cuadro 14: Matriz de Confusión, modelo para MSFT usando redes neuronales

\begin{tabular}{|c|c|c|c|}
\hline & \multicolumn{2}{|c|}{ Clase Real } \\
\hline & & Down & Up \\
\hline \multirow{2}{*}{ Predicción } & Down & 5 & 3 \\
\hline & $\mathrm{Up}$ & 3 & 11 \\
\hline
\end{tabular}

Elaboración propia.

La matriz de confusión muestra como el modelo logra predecir correctamente 11 variaciones porcentuales positivas de la acción y 5 negativas, logrando una precisión del $71.91 \%$ sobre los datos de validación.

\section{Conclusiones}

Para el modelo NLP de etiquetado automático de los textos, los resultados de los tres predictores se resumen en el cuadro 15 .

Cuadro 15: Resultados: Predictores para modelo NLP

\begin{tabular}{|c|c|c|c|}
\cline { 2 - 4 } \multicolumn{1}{c|}{} & \multicolumn{3}{c|}{ Modelos } \\
\hline Medidas de Rendimiento & Random Forest & XGBoost & Red Neuronal \\
\hline Precisión Durante Validación & 0.754637 & $\mathbf{0 . 8 0 0 0}$ & 0.7549 \\
\hline Precisión Durante Test & $\mathbf{0 . 7 5 3 0 2}$ & $\mathbf{0 . 7 5 3 0 2}$ & 0.75293 \\
\hline AUC & $\mathbf{0 . 5 7}$ & $\mathbf{0 . 5 7}$ & $\mathbf{0 . 5 7}$ \\
\hline
\end{tabular}

Elaboración propia.

Según el cuadro, todos los modelos logran un AUC idéntico, y los modelos basados en árboles lograron la misma precisión durante la validación. Es por esto que el mejor modelo para el etiquetado automático de los textos es el de XGBoost, dado que logró una precisión promedio durante la validación superior a la de los demás modelos.

A pesar de ser el mejor en este caso, se debe tener en cuenta los resultados de la matriz de confusión. Según el cuadro 4, el modelo es bastante eficiente al identificar los textos que hablan de tendencias alcistas, lo que explica la precisión del modelo. Sin embargo, no es muy bueno ante la identificación de textos que hablan de una tendencia a la baja de del precio de la acción, obteniendo una alta tasa de falsos positivos.

Aunque la investigación pretende estudiar estrategias de aprovechamiento de la información proveniente de StockTwits, esta es una aproximación inicial y como trabajo futuro; sería ideal probar con otro modelo de procesamiento del lenguaje natural, que aproveche un poco más la información extraída de los textos.

Sobre la utilización de la información de la red social para la predicción de la dirección de la variación de los precios de los activos, en los cuadros 16, 17 y 18 se resumen las medidas de rendimiento de los predictores propuestos para cada una de las acciones de interés.

El cuadro 16 permite evidenciar que el predictor Random Forest, logra las mejores medidas de rendimiento para predecir la dirección de la variación del precio de la acción de Apple Inc. Dada la matriz de confusión del cuadro 6, se observa como; a pesar del tamaño de muestra para la validación; no hay un número elevado de falsos positivos (solo 2 variaciones negativas del precio etiquetadas como positivas). 
Cuadro 16: Resultados: Predictores para precio de Apple Inc.

\begin{tabular}{|c|c|c|c|}
\cline { 2 - 4 } \multicolumn{1}{c|}{} & \multicolumn{3}{c|}{ Apple Inc. } \\
\hline Medidas de Rendimiento & Random Forest & XGBoost & Red Neuronal \\
\hline Precisión Durante Validación & $\mathbf{1 . 0 0 0}$ & $\mathbf{1 . 0 0 0}$ & 0.880 \\
\hline Precisión Durante Test & $\mathbf{0 . 7 7 2 7}$ & 0.7272 & 0.5910 \\
\hline AUC & $\mathbf{0 . 6 0}$ & 0.45 & 0.53 \\
\hline
\end{tabular}

Elaboración propia.

Para la acción de IBM, el mejor modelo es también el de Random Forest. A pesar de que no logra la tasa más alta de precisión durante el test, si lo hace durante la validación y el área bajo la curva es mayor que la de los demás predictores. A pesar de esto, para esta acción; el modelo tiene una debilidad, pues según su matriz de confusión sobre los datos de test (cuadro 9) el modelo predice en esa pequeña muestra 10 variaciones negativas del precio como positivas, lo cual explica las zonas de la curva ROC debajo de la línea de incertidumbre.

\begin{tabular}{|c|c|c|c|}
\hline & \multicolumn{3}{|c|}{ IBM } \\
\hline Medidas de Rendimiento & Random Forest & XGBoost & Red Neuronal \\
\hline Precisión Durante Validación & 1.000 & 1.000 & 0.9300 \\
\hline Precisión Durante Test & 0.3636 & 0.4090 & 0.4091 \\
\hline AUC & 0.54 & 0.50 & 0.51 \\
\hline
\end{tabular}

Elaboración propia.

Sobre las acciones de Microsoft, el cuadro 18 indica que los candidatos a mejor modelo para este activo son del de XGBoost y el de Redes Neuronales, de los cuales se selecciona como ideal el primero. A pesar de que la red neuronal tiene tasas de precisión altas durante la validación y la mayor tasa de precisión sobre la muestra de test, el modelo XGBoost; tiene una mayor área bajo la curva y un mejor comportamiento de su curva ROC, evidenciando un predictor balanceado y estable.

Cuadro 18: Resultados: Predictores para precio de Microsoft

\begin{tabular}{|c|c|c|c|}
\cline { 2 - 4 } \multicolumn{2}{c|}{} & \multicolumn{3}{c|}{ Microsoft } \\
\hline Medidas de Rendimiento & Random Forest & XGBoost & Red Neuronal \\
\hline Precisión Durante Validación & $\mathbf{1 . 0 0 0}$ & $\mathbf{1 . 0 0 0}$ & 0.890 \\
\hline Precisión Durante Test & 0.5909 & 0.6363 & $\mathbf{0 . 7 1 9 1}$ \\
\hline AUC & 0.64 & $\mathbf{0 . 8 6}$ & 0.74 \\
\hline
\end{tabular}

Elaboración propia.

En general el rendimiento de los modelos ante el problema de predecir la dirección de la variación del precio no es el ideal de un predictor estable, balanceado y con altas tasas de precisión. Sin embargo, esto no implica afirmar que la información de la red social no tenga poder predictivo alguno. De los resultados de esta investigación se tiene que es posible usar este recurso para este propósito, y como trabajo futuro; se pueden buscar otras fuentes para potenciarlo (entre ellas la descarga de un set de datos con información de un periodo de tiempo más amplio, descargas de datos más frecuentes durante el día, variables adicionales, entre otras). 


\section{Referencias}

Asur, S., \& Huberman, B. A. (2010, August). Predicting the future with social media. In Proceedings of the 2010 IEEE/WIC/ACM International Conference on Web Intelligence and Intelligent Agent Technology-Volume 01 (pp. 492-499). IEEE Computer Society.

Beysolow II, T. Applied Natural Language Processing with Python.

Copestake, A. (2005). Natural language processing. Lecture Notes, Computer Laboratory, University of Cambridge.

Coyne, S., Madiraju, P., \& Coelho, J. (2017, November). Forecasting Stock Prices Using Social Media Analysis. In Dependable, Autonomic and Secure Computing, 15th Intl Conf on Pervasive Intelligence \& Computing, 3rd Intl Conf on Big Data Intelligence and Computing and Cyber Science and Technology Congress (DASC/PiCom/DataCom/CyberSciTech), 2017 IEEE 15th Intl (pp. 1031-1038). IEEE.

Dale, R., Moisl, H., \& Somers, H. (2000). Handbook of natural language processing. CRC Press.

Feldman, R. (2013). Techniques and applications for sentiment analysis. Communications of the ACM, 56(4), 82-89.

Friedman, J. H. (2001). Greedy function approximation: a gradient boosting machine. Annals of statistics, 1189-1232.

Friedman, J. H. (2002). Stochastic gradient boosting. Computational Statistics \& Data Analysis, $38(4), 367-378$.

Friedman, J., Hastie, T., \& Tibshirani, R. (2000). Additive logistic regression: a statistical view of boosting (with discussion and a rejoinder by the authors). The annals of statistics, 28(2), 337-407.

Friedman, J. H., \& Popescu, B. E. (2003). Importance sampled learning ensembles. Journal of Machine Learning Research, 94305.

Hebb, D. O. (1949). The organization of behavior.

Izenman, A. J. (2008). Modern multivariate statistical techniques. Regression, classification and manifold learning.

Jurafsky, D. (2000). Speech and language processing: An introduction to natural language processing. Computational linguistics, and speech recognition.

Kimoto, T., Asakawa, K., Yoda, M., \& Takeoka, M. (1990, June). Stock market prediction system with modular neural networks. In Neural Networks, 1990., 1990 IJCNN International Joint Conference on (pp. 1-6). IEEE. 
Kooijman, J. F. (2014). Stock market prediction using social media data and finding the covariance of the LASSO.

LeBaron, B. (2006). Agent-based computational finance. Handbook of computational economics, 2, $1187-1233$.

McCulloch, W. S., \& Pitts, W. (1943). A logical calculus of the ideas immanent in nervous activity. The bulletin of mathematical biophysics, 5(4), 115-133.

Marquez, L. (2000). Machine learning and natural language processing. In Complementary documentation for the conference "Aprendizaje automático aplicado al procesamiento del lenguaje natural", Soria.

McEnery, T., Xiao, R., \& Tono, Y. (2006). Corpus-based language studies: An advanced resource book. Taylor \& Francis.

Nausheen, S., Kumar, A., \& Amrutha, K. K. SURVEY ON SENTIMENT ANALYSIS OF STOCK MARKET. ISO 690

Oh, C., \& Sheng, O. (2011, December). Investigating Predictive Power of Stock Micro Blog Sentiment in Forecasting Future Stock Price Directional Movement. In Icis (pp. 1-19).

Oliveira, N., Cortez, P., \& Areal, N. (2013, September). On the predictability of stock market behavior using stocktwits sentiment and posting volume. In Portuguese Conference on Artificial Intelligence (pp. 355-365). Springer, Berlin, Heidelberg.

Samanidou, E., Zschischang, E., Stauffer, D., \& Lux, T. (2007). Agent-based models of financial markets. Reports on Progress in Physics, 70(3), 409.

Theeramunkong, T., Kongkachandra, R., \& Supnithi, T. Advances in Natural Language Processing, Intelligent Informatics and Smart Technology.

Tsui, D. Predicting Stock Price Movement Using Social Media Analysis. Stanford University, Technical Report.

Werbos, P.J. (1974). Beyond regression: new tools for prediction and analysis in the behavioral sciences, Ph.D. dissertation, Harvard University.

Zhang, X., Fuehres, H., \& Gloor, P. A. (2011). Predicting stock market indicators through twitter "I hope it is not as bad as I fear". Procedia-Social and Behavioral Sciences, 26, 55-62. 
Bogotá, 3 de diciembre de 2018

\section{Referencia}

Habeas Data Trabajo de Grado

\section{Señores}

Comité de Opción de Grado

Facultad de Estadística

Universidad Santo Tomás

Cordial saludo,

Por medio de la presente informo que los datos utilizados para este estudio fueron obtenidos a través de las plataformas "StockTwits for Developers" y "Financial \& Alternative Data - Quand". Información que se encuentra abierta para uso público y cada una de las plataformas provee soporte y manuales para su acceso, por lo cual se tiene autorización para su divulgación.

\footnotetext{
Alf

Andrés Felipe Rodriguez Perez

Estudiante

Facultad de Estadística

Universidad Santo Tomás
} 